\title{
Hydrodynamic Interactions between Two Forced Objects of Arbitrary Shape: I Effect on Alignment
}

Tomer Goldfriend, 1, (a) Haim Diamant, 2, b) and Thomas A. Witten,

1) Raymond \& Beverly Sackler School of Physics and Astronomy, Tel Aviv University, Tel Aviv 69978, Israel

2) Raymond \&3 Beverly Sackler School of Chemistry, Tel Aviv University, Tel Aviv 69978, Israel

3) Department of Physics and James Franck Institute, University of Chicago, Chicago, Illinois 6063\%, USA

(Dated: 3 May 2016)

We study the properties and symmetries governing the hydrodynamic interaction between two identical, arbitrarily shaped objects, driven through a viscous fluid. We treat analytically the leading (dipolar) terms of the pair-mobility matrix, affecting the instantaneous relative linear and angular velocities of the two objects at large separation. We prove that the instantaneous hydrodynamic interaction linearly degrades the alignment of asymmetric objects by an external time-dependent drive [Moths and Witten, Phys. Rev. Lett. 110, 028301 (2013)]. The time-dependent effects of hydrodynamic interactions are explicitly demonstrated through numerically calculated trajectories of model alignable objects composed of four stokeslets. In addition to the orientational effect, we find that the two objects usually repel each other. In this case the mutual degradation weakens as the two objects move away from each other, and full alignment is restored at long times.

PACS numbers: 47.57.ef, 47.57.J-, 47.63.mf, 82.70.Dd, 05.45.-a

\footnotetext{
a) Electronic mail: goldfriend@tau.ac.il

b) Electronic mail: hdiamant@tau.ac.il

c) Electronic mail: t-witten@uchicago.edu
} 


\section{INTRODUCTION}

The dynamics of colloid suspensions is crucially influenced by flow-mediated correlations ${ }^{12 !}$. While these hydrodynamic interactions (HI) have an important role in the dynamics of ambient suspensions at thermal equilibrium ${ }^{2}$, their effect becomes even more pronounced for objects driven out of equilibrium, where the total force acting on each object generates a long-ranged flow, decaying as $1 / R$ with the distance $R$ between the objects. A well-known example is colloid sedimentation, where HI lead to strongly correlated motions and largescale dynamic structures ${ }^{3}$. Various types of driving, such as electrophoresis, are widely used to control the transport of colloids and other polyatomic objects ${ }^{2}$. Theoretical studies of driven colloids traditionally focus on regular particle shapes such as uniform spheres and ellipsoids. The driving of more asymmetric objects is richer ${ }^{4 / 7}$ as it generally includes coupling between translation and rotation - when the object is subjected to a force it also rotates, and when it is under torque it also translates ${ }^{1}$. The choice of a rotation sense under a unidirectional force implies a chiral response of the driven object. Such richer responses can be exploited to obtain "steerable colloids" - objects whose orientation and transport can be controlled in much more detail. For example, applying a torque by a rotating uniform magnetic field was used to achieve efficient transport of chiral magnetic objects ${ }^{8}$. Another example, which is the main issue of the present work, is the ability to achieve orientational alignment of asymmetric objects by applying an external force 911 .

The earlier theoretical works of Refs. ${ }^{[11}$ dealt with isolated asymmetric objects in Stokes flow, which exhibit a chiral response. The object's chiral response is encoded in the offdiagonal block of its self-mobility matrix, referred to as the twist matrix. Some objects have a twist matrix that leads them to align one axis in the body with the applied force. If the twist matrix has only a single real eigenvalue, the object becomes "axially aligned" in this way ${ }^{\sqrt{5}}$, and the aligning direction is along the corresponding eigenvector. Hence, in the absence of HI and thermal fluctuations, a set of identical, axially aligning objects reach a partially aligned state, where all the objects rotate about the same axis with the same angular velocity, but with an arbitrary phase. Furthermore, it was shown that, by applying an appropriate time-dependent forcing, the system can be driven to a fully aligned state, where all the objects are phase-locked with the force and rotate in synchrony 10111 .

In view of the above we use throughout this article the following terminology concerning 
the response of various objects: (i) symmetric objects (such as a uniform sphere); (ii) regular objects, which are asymmetric objects with a vanishing twist matrix (such as a uniform ellipsoid); (iii) irregular objects, having a non-vanishing twist matrix; (iv) axially alignable objects, which are irregular objects, whose twist matrix has a single real eigenvalue. We note that the twist matrix depends on the position of the forcing point as well. For example, an ellipsoid whose forcing point is displaced from its centroid, i.e., an ellipsoid with a nonuniform mass distribution under gravity, has a non-vanishing twist matrix, and generally might be alignable.

The theoretical groundwork for treating the HI between arbitrary objects in Stokes flow was laid by Brenner and O'Neil12213. The theory was subsequently applied to a pair of particles of various regular shapes ${ }^{1420}$. To this one should add many earlier studies of the collective dynamics of suspensions made of ellipsoids1-25. We note that there are key differences between asymmetric objects, such as ellipsoids, and the irregular objects studied here. The symmetries of a uniform ellipsoid lead to: (a) the absence of a translationrotation coupling for a single object, and therefore lack of alignability; (b) the absence of a $1 / R^{2}$ contribution to the relative velocity developed between two such objects at mutual distance $R$. Finally, several numerical techniques have been introduced to treat suspensions of arbitrarily shaped objects $56 \mid 30$.

In this work we focus on simple, general properties of the pair HI between two arbitrarily shaped objects at zero Reynolds number, and the resulting effect on their orientational alignment. The study of translational effects will be presented in a separate publication.

The work is made of two distinct parts. The first part treats rigorously the instantaneous hydrodynamic interaction, i.e., the pair-mobility matrix. We use Brenner's analytical framework ${ }^{[3132}$, specializing to the leading order of the $\mathrm{HI}$ in the distance between the objects (multipole expansion, also known as the method of reflections ${ }^{1}$ ). The second part addresses the time-dependent trajectories of forced objects. This is a multi-variable, highly non-linear dynamical system exhibiting complex and diverse dynamics. In this part we are limited to numerical integration of the objects' trajectories. We provide typical examples for the time evolution of pairs of stokeslet objects.

We begin by discussing in Sec. II the general properties and symmetries of the pairmobility matrix for two arbitrarily shaped objects. In Sec. III we apply a multipole expansion to the pair-mobility matrix and obtain results for the instantaneous HI at large distances. 
In Sec. IV we derive the resulting properties of stokeslet objects, and in Sec. $\mathrm{V}$ we use them to perform numerical time integration for the evolution of object pairs and their alignment. Finally, in Sec. VI, we discuss several consequences of our results.

\section{PAIR-MOBILITY MATRIX: GENERAL CONSIDERATIONS}

\section{A. Structure of the Pair-Mobility Matrix}

The kinematics of a rigid object is represented by a translational velocity $\vec{V}$, which refers to an arbitrary reference point rigidly affixed to the object, and an angular velocity $\vec{\omega}$. We designate the reference point as the origin of the object. Note that the angular velocity of the object is independent of the choice of its origin, and that the origin does not necessarily lie on the instantaneous axis of rotation of the object.

Consider two arbitrarily shaped rigid objects, $a$ and $b$, with typical size $l$, subject to external forces and torques $\vec{F}^{a}, \vec{F}^{b}$ and $\vec{\tau}^{a}, \vec{\tau}^{b}$ in an unbounded, otherwise quiescent fluid of viscosity $\eta$. In the creeping flow regime, the objects respond with linear and angular velocities to the external forces and torques through a $12 \times 12$ pair-mobility matrix,

$$
\left(\begin{array}{l}
\overrightarrow{\mathcal{V}}^{a} \\
\overrightarrow{\mathcal{V}}^{b}
\end{array}\right)=\frac{1}{\eta l}\left(\begin{array}{ll}
\mathbb{M}^{a a} & \mathbb{M}^{a b} \\
\mathbb{M}^{b a} & \mathbb{M}^{b b}
\end{array}\right)\left(\begin{array}{c}
\overrightarrow{\mathcal{F}}^{a} \\
\overrightarrow{\mathcal{F}}^{b}
\end{array}\right),
$$

where we define generalized velocity and generalized force 6-vectors, $\overrightarrow{\mathcal{V}}^{x}=\left(\vec{V}^{x}, l \vec{\omega}^{x}\right)^{T}$ and $\overrightarrow{\mathcal{F}}^{x}=\left(\vec{F}^{x}, \overrightarrow{\tau^{x}} / l\right)^{T}$ for $x=a, b$. The diagonal blocks, $\mathbb{M}^{a a}$ and $\mathbb{M}^{b b}$, correspond to the selfmobilities of the objects (which nevertheless depend on the configuration of both objects). The off-diagonal blocks, $\mathbb{M}^{a b}$ and $\mathbb{M}^{b a}$, describe the pair hydrodynamic interaction. We hereafter omit the factor $(\eta l)^{-1}$ (i.e., set $\eta l=1$ ). This, together with the representation of the generalized forces and velocities, make $\mathbb{M}$ dimensionless and dependent on the geometry alone. Throughout the text we designate 6-vectors and matrices with calligraphic font and blackboard-bold letters, respectively. A detailed description of the notation used in the article is given in Appendix A.

Since $\vec{V}$ and $\vec{\tau}$ depend on the choice of object origins, so does the pair-mobility matrix. The transformation between pair-mobility matrices corresponding to different origins is given in Appendix B. 
The pair-mobility matrix is a function of the objects' geometries, their orientations, and the vector connecting their origins, indicated hereafter by $\vec{R}$. (We define the direction of $\vec{R}$ from the origin of object $b$ to the origin of object $a$.) The geometry of object $x$ is denoted by $\boldsymbol{r}^{x}$. For example, if the object consists of a discrete set of $N_{x}$ stokeslets (see Sec. IV A), then $\boldsymbol{r}^{x}$ is a $3 N_{x}$-vector specifying the positions of the stokeslets; otherwise, it represents the surface of the object.

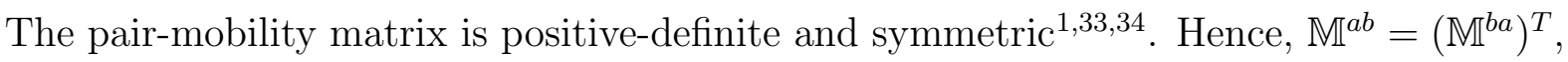
and the self-blocks can be written as

$$
\mathbb{M}^{x x}=\left(\begin{array}{cc}
\mathbb{A}^{x x} & \left(\mathbb{T}^{x x}\right)^{T} \\
\mathbb{T}^{x x} & \mathbb{S}^{x x}
\end{array}\right)
$$

As in the analysis for isolated objects ${ }^{9}$, the self-mobility matrix contains the following $3 \times 3$ blocks: the alacrity matrix $\mathbb{A}$ (translational response to force); the screw matrix $\mathbb{S}$ (rotational response to torque); and the twist matrix $\mathbb{T}$ (translation-rotation coupling). The twist matrix characterizes the chiral response of the object (the sense of rotation under a force). In the present article we deal with alignable objects, whose individual $\mathbb{T}$ is necessarily nonvanishing. Furthermore, in the case of a pair of objects, the presence of the other object makes the self-twist matrix, $\mathbb{T}^{x x}$, differ from the single-object one. As to the off-diagonal blocks of the pair-mobility matrix, the symmetry of $\mathbb{M}$ implies the following structure:

$$
\mathbb{M}^{a b}=\left(\begin{array}{cc}
\mathbb{A}^{a b} & \left(\mathbb{T}^{b a}\right)^{T} \\
\mathbb{T}^{a b} & \mathbb{S}^{a b}
\end{array}\right), \quad \mathbb{M}^{b a}=\left(\begin{array}{cc}
\left(\mathbb{A}^{a b}\right)^{T} & \left(\mathbb{T}^{a b}\right)^{T} \\
\mathbb{T}^{b a} & \left(\mathbb{S}^{a b}\right)^{T}
\end{array}\right)
$$

\section{B. Further Symmetries of the Pair-Mobility Matrix}

The discussion in the preceding subsection has been for a general pair of objects, which are not necessarily identical. In the present subsection, we focus on the case in which the two objects are identical in shape and orientation, i.e., $\boldsymbol{r}^{a}=\boldsymbol{r}^{b} \equiv \boldsymbol{r}$. Our goal is to understand what the instantaneous relative velocities (linear and angular) between the two objects are, when the objects are subjected to the same external forcing. The restriction to identical objects makes $\mathbb{M}$ invariant under exchange of objects. This additional symmetry is made of two operations: interchanging the blocks $\mathbb{M}^{a a} \leftrightarrow \mathbb{M}^{b b}$ and $\mathbb{M}^{a b} \leftrightarrow \mathbb{M}^{b a}$; and inversion of $\vec{R}$. That is,

$$
\mathbb{M}(\boldsymbol{r}, \vec{R})=\mathbb{E} \mathbb{M}(\boldsymbol{r},-\vec{R}) \mathbb{E}^{-1}
$$


where $\mathbb{E}$ is a $12 \times 12$ matrix which interchanges the objects,

$$
\mathbb{E}=\left(\begin{array}{cc}
0 & \mathbb{I}_{6 \times 6} \\
\mathbb{I}_{6 \times 6} & 0
\end{array}\right)
$$

with $\mathbb{I}_{6 \times 6}$ denoting the $6 \times 6$ identity matrix.

The symmetry to object exchange, when combined with the parity of $\mathbb{M}$ (i.e., whether it remains the same or changes sign) under $\vec{R}$-inversion,,$\sqrt[35]{,}$ has important consequences for the effect of hydrodynamic interactions on alignment. If $\mathbb{M}$ has a definite parity one can determine what the relative response of the objects to forcing is - i.e., whether they attain the same or the opposite linear and angular velocities. If the term is symmetric to inversion, the velocities would be identical, and if it is antisymmetric, they would be opposite. This is because

$$
\left(\begin{array}{ll}
\mathbb{M}^{a a}(\vec{R}) & \mathbb{M}^{a b}(\vec{R}) \\
\mathbb{M}^{b a}(\vec{R}) & \mathbb{M}^{b b}(\vec{R})
\end{array}\right)= \pm\left(\begin{array}{cc}
\mathbb{M}^{a a}(-\vec{R}) & \mathbb{M}^{a b}(-\vec{R}) \\
\mathbb{M}^{b a}(-\vec{R}) & \mathbb{M}^{b b}(-\vec{R})
\end{array}\right)= \pm\left(\begin{array}{cc}
\mathbb{M}^{b b}(\vec{R}) & \mathbb{M}^{b a}(\vec{R}) \\
\mathbb{M}^{a b}(\vec{R}) & \mathbb{M}^{a a}(\vec{R})
\end{array}\right)
$$

where the second equality comes from the response to exchange of objects, Eq. (2). Consequently, under identical forcing of the two objects one finds,

$$
\overrightarrow{\mathcal{V}}^{a}=\left(\mathbb{M}^{a a}+\mathbb{M}^{a b}\right) \overrightarrow{\mathcal{F}}= \pm\left(\mathbb{M}^{b b}+\mathbb{M}^{b a}\right) \overrightarrow{\mathcal{F}}= \pm \overrightarrow{\mathcal{V}}^{b}
$$

Thus, since any $\mathbb{M}$ can be decomposed into even and odd terms, we find that only the odd ones cause relative motions of the two objects.

The pair-mobility as a whole, however, never has a definite parity under $\vec{R}$-inversion, i.e., it is made of both even and odd terms. This becomes clear when $\mathbb{M}(\boldsymbol{r}, \vec{R})$ is expanded in small $l / R$, i.e., in multipoles. A general discussion of the parity of each multipole term is given in the next section. For now, let us consider those two leading multipoles which are independent of the objects' shape, and therefore always exist. The monopole-monopole interaction (Oseen tensor), which is the leading term in $\mathbb{A}^{a b}$ making particle $a$ translate due to the force on particle $b$, is symmetric under $\vec{R}$-inversion. The part of the monopole-dipole interaction causing the second object to rotate due to the force on the first, i.e., the leading term in $\mathbb{T}^{a b}$, is antisymmetric. For example, even the most symmetric pair of objects - two spheres - has an $\vec{R}$-symmetric $\mathbb{A}^{a b}$, leading to zero relative velocity, and an $\vec{R}$-antisymmetric $\mathbb{T}^{a b}$, causing them to rotate with opposite senses ${ }^{1}$. Thus, for a general object, the highest 
order which maintains $\mathbb{M}$ of definite parity is the monopole $1 / R$ Oseen one, which is even. (The self-blocks are constant up to order $1 / R^{4}$; see below.)

From this discussion we can immediately conclude that, to leading order in the separation of two identical, fully aligned objects, their instantaneous hydrodynamic interaction must linearly degrade the alignment. The leading degrading term comes from $\mathbb{T}^{a b}$, their rotational response to force, and is of order $1 / R^{2}$. It is worthwhile to note again that such a rotational response is present as well for a pair of uniform spheres or ellipsoids; yet, such regular objects are not alignable to begin with.

The relation between object-exchange symmetry and the symmetry of the linear-velocity response is intimately related to the issue of hydrodynamic pseudo-potentials $\sqrt{36}$, which will be discussed in detail in a forthcoming publication.

\section{FAR-FIELD INTERACTION: MULTIPOLE EXPANSION}

There are two characteristic length scales in our problem: the typical size of the objects, $l$, and the distance between them, $R=|\vec{R}|$. If $l \ll R$, we can write the pair-mobility matrix as a power series in $(l / R)$,

$$
\mathbb{M}=\mathbb{M}_{(0)}+\mathbb{M}_{(1)}+\mathbb{M}_{(2)}+\ldots
$$

where $\mathbb{M}_{(n)} \sim(l / R)^{n}$. The analysis of this expansion as given below holds for any pair of objects, whether identical or not. The zeroth order, $\mathbb{M}_{(0)}$, is a block diagonal matrix which is made of the self-mobilities of the two non-interacting objects. (These should be distinguished from $\mathbb{M}^{a a}$ and $\mathbb{M}^{b b}$, the self-mobilities of the interacting objects.)

The hydrodynamic multipole expansion (also known as the method of reflections) is based on the Green's function of Stokes flow, the Oseen tensor ${ }^{1}$, given in our units $(\eta l=1)$ by

$$
\mathbb{G}_{i j}(\vec{r})=\frac{1}{8 \pi} \frac{l}{r}\left(\delta_{i j}+\frac{r_{i} r_{j}}{r^{2}}\right)
$$

which is a symmetric $3 \times 3$ tensor, invariant under $\vec{r}$-inversion. A point force at $\vec{r}_{0}, \delta\left(\vec{r}-\vec{r}_{0}\right) \vec{f}$, generates a velocity field $\vec{u}(\vec{r})=\mathbb{G}\left(\vec{r}-\vec{r}_{0}\right) \cdot \vec{f}$.

We obtain two general results concerning the multipoles of the hydrodynamic interaction between two arbitrary objects. The two objects need not be identical. The proofs are given in Appendix $\mathrm{D}, 37$ 
1. The leading interaction multipole in the self-blocks of the pair-mobility matrix is $n=4$. That is, any response of one object to forces on itself, owing to the other object, must fall off with distance $R$ between the objects at least as fast as $R^{-4}$.

2. The nth multipole has self-blocks of $(-1)^{n}$ parity, and coupling blocks of the opposite, $(-1)^{n+1}$ parity. Thus, e.g., the leading term in $\mathbb{M}^{a a}$, proportional to $R^{-4}$, is invariant under $\vec{R}$-inversion, and the $R^{-4}$ part of $\mathbb{M}^{a b}$ changes sign under $\vec{R}$-inversion. Likewise for the multipole varying as $\vec{R}^{-5}$, the $\mathbb{M}^{a a}$ changes sign under $\vec{R}$-inversion while $\mathbb{M}^{a b}$ remains invariant. $\underline{35}$

These statements pertain to the mobility matrix. As to the propulsion matrix (the inverse of the mobility matrix), the leading correction to the self-block becomes $\sim 1 / R^{2}$, and the second statement concerning parity remains intact.

We now consider for a moment two identical objects and specialize to the first and second multipoles, i.e., the hydrodynamic interaction up to order $1 / R^{2}$. The discussion in the preceding and current sections implies the following form of the two leading terms in the pair-mobility matrix:

$$
\mathbb{M}_{(1)}=\left(\begin{array}{cc}
0 & \mathbb{M}_{(1)}^{a b} \\
\mathbb{M}_{(1)}^{a b} & 0
\end{array}\right), \quad \mathbb{M}_{(2)}=\left(\begin{array}{cc}
0 & \mathbb{M}_{(2)}^{a b} \\
-\mathbb{M}_{(2)}^{a b} & 0
\end{array}\right)
$$

In more detail: there are no first- and second-order corrections to the objects' self-mobility. Hence, these two multipoles have definite parities - the first is even, and the second is odd. Consequently, the first multipole does not cause any relative motion of the two objects, whereas the second mutipole makes them translate and rotate in opposite linear and angular velocities.

The essential characteristics of the first two multipoles are schematically illustrated in Fig. 1. The first multipole arises directly from the Green's function,

$$
\mathbb{M}_{(1)}^{a b}=\left(\begin{array}{cc}
\mathbb{G}(\vec{R}) & 0 \\
0 & 0
\end{array}\right)
$$

where $\mathbb{G}(\vec{R})$ is the Oseen tensor, given in Eq. (5).

In the interaction described by the second multipole one object sees the other as a point, see Fig. 1. Accordingly, this term contains two types of interaction: (1) the response of object $a$ to the non-uniformity of the flow due to the force monopole at object $b$ (regarded 
as a point); (2) the advection of object $a$ (regarded as a point) by the flow due to the force dipole acting at object $b$. These two effects are both proportional to $\vec{\nabla} \mathbb{G}(\vec{R}) \sim 1 / R^{2}$. Each can be written as a product of a tensor which arises from the medium alone, through derivatives of the Oseen tensor $\vec{\nabla} \mathbb{G}(\vec{R})$, and another tensor which depends on the objects' geometry. The second-order correction to the velocity of object $a$ is given by the sum of these two effects, each expressed in terms of a coupling tensor $\Theta$ and an object tensor $\Phi$

$$
\begin{aligned}
\overrightarrow{\mathcal{V}}_{(2)}^{a} & =\mathbb{M}_{(2)}^{a b} \cdot \overrightarrow{\mathcal{F}}^{b} \\
\mathbb{M}_{(2)}^{a b} & =\Phi^{a}: \Theta(\vec{R})-\Theta^{T}(\vec{R}): \tilde{\Phi}^{b}
\end{aligned}
$$

where the double dot notation denotes a contraction over two indices. Equation (8) contains three tensors of rank 3, denoted by capital Greek letters. The first, $\Phi$, with dimensions $6 \times 3 \times 3$, gives the generalized velocity of the object in linear response to the velocity gradient of the flow in which it is embedded. The second, $\tilde{\Phi}$, having dimensions $3 \times 3 \times 6$, gives the force dipole acting on the fluid around the object's origin in linear response to the generalized force acting on it. Both $\Phi$ and $\tilde{\Phi}$ depend on the objects' geometry alone ${ }^{38}$. The third tensor, $\Theta$, with dimensions $3 \times 3 \times 6$, describes the coupling of these object responses through the fluid. It is given by

$$
\Theta_{s k j}(\vec{R}) \equiv \begin{cases}\left.\partial_{s} \mathbb{G}_{k j}(\vec{r})\right|_{\vec{R}} & j=1,2,3 \\ 0 & j=4,5,6\end{cases}
$$

Repeating the same procedure for $\overrightarrow{\mathcal{V}}^{b}$ in response to $\overrightarrow{\mathcal{F}}^{a}$ while using the odd parity of $\Theta$, we get

$$
\mathbb{M}_{(2)}^{b a}=\Theta^{T}(\vec{R}): \tilde{\Phi}^{a}-\Phi^{b}: \Theta(\vec{R})
$$

The tensors $\Phi$ and $\tilde{\Phi}$ are not independent $\frac{39}{3}$. We now show that $\Phi=\tilde{\Phi}^{T}$. The symmetry of $\mathbb{M}$ implies that each multipole is also a symmetric matrix. Using Eqs. (8) and (10) and equating $\left(\mathbb{M}_{(2)}^{b a}\right)^{T}=\mathbb{M}_{(2)}^{a b}$, we get $\tilde{\Phi}^{a}=\left(\Phi^{a}\right)^{T}$ and $\tilde{\Phi}^{b}=\left(\Phi^{b}\right)^{T}$.

To summarize, the matrix $\mathbb{M}_{(2)}$ is given by

$$
\mathbb{M}_{(2)}=\left(\begin{array}{cc}
0 & \Phi^{a}: \Theta(\vec{R})-\left[\Phi^{b}: \Theta(\vec{R})\right]^{T} \\
-\Phi^{b}: \Theta(\vec{R})+\left[\Phi^{a}: \Theta(\vec{R})\right]^{T} & 0
\end{array}\right) .
$$

This results is valid for a general pair of objects. If the two objects are identical, the offdiagonal blocks have the same form with opposite signs. The additional condition that the entire $\mathbb{M}$ must be symmetrical implies then that each block by itself is antisymmetric. 


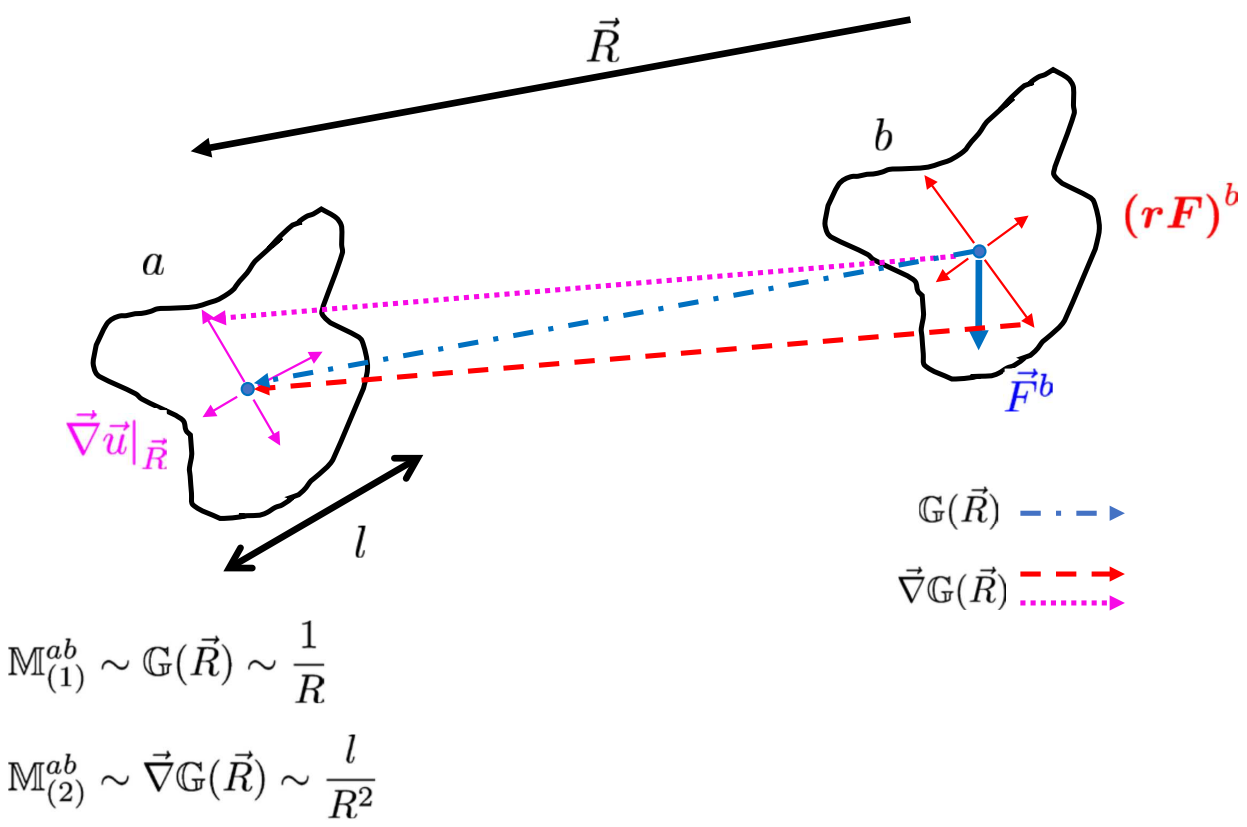

FIG. 1. Illustration of the two leading orders of the hydrodynamic interaction between two forced objects. The leading term in the pair-mobility matrix (light blue/dash-dotted arrow between the objects' origins), decaying as $1 / R$, comes from the point-like response of object $a$ to the local flow caused by the force monopole on object $b$ (blue/thick arrow). The next-order term, decaying as $l / R^{2}$, has two contributions: (i) The point-like response of object $a$ to the local flow caused by the force dipole on object $b$ (red/dashed arrow from the red/thin arrows at object $b$ to the origin of a). (ii) The response of object $a$ to the local flow gradient caused by the force monopole on object $b$ (magenta/dotted arrow from the origin of $b$ to the magenta/thin arrows at object $a$ ).

By separating the tensors $\Phi$ and $\Theta$ into their symmetric and antisymmetric parts, the second-order term of the pair-mobility matrix can be simplified further. It should be mentioned, in addition, that the $\Phi$ tensor depends on the origin selected for the object. These two technical issues are addressed in Appendices E and C, respectively. Finally, we note that the terms in these tensors corresponding to the translational response vanish for spheres and ellipsoids. Consequently, two such regular objects develop relative velocity only to orders $1 / R^{3}$ and above. 


\section{NUMERICAL ANALYSIS FOR STOKESLET OBJECTS}

In the preceding sections we have derived the general properties of the instantaneous hydrodynamic interaction between two arbitrarily shaped objects. We now move on to the second part of the work, addressing the time evolution of the two objects. This complicated problem is not tractable analytically, and we resort to numerical integration of specific examples. Because of the complexity of the problem, and since we are interested in generic properties, we allow ourselves to restrict the analysis to the simplest, even if unrealistic, objects. Arguably the simplest form of an arbitrarily shaped object is the so-called stokeslet object - a discrete set of small spheres, separated by much larger, rigid distances, where each sphere is approximated as a point force. The sparseness of these objects makes them free-draining, which may be valid for macromolecules but not for compact objects.

We treat pairs of identical objects, each made of four stokeslets. To obtain representative sampling of numerical examples we do not design these objects but create them randomly. Four points are placed at random distances ranging between 0 and 1 from an arbitrary origin. The origin is then shifted to the points' center of mass. The radius $\rho$ of the stokeslets is taken as 0.01 . The resulting configuration is checked to be "sufficiently chiral", in the sense that the $\mathbb{T}$-matrix of the individual object is strongly asymmetric, having a single real eigenvalue of absolute value $\left|\lambda_{3}\right|>0.005$, which makes the object axially alignable. (See Sec. If). Examples of the stokeslet objects we use are provided in Fig. 2 .

The way to calculate the mobility of a single stokeslet object was presented in Ref. 9. First, we briefly present in Sec. IVA the simple extension of this method to pair-mobilities. We calculate both the pair mobility and the tensor $\Phi$ introduced in Secs. [I] and III. The latter allows us to calculate pair mobilities up to second order in the multipole expansion. Section IVB describes how we use the pair mobility to numerically calculate the time evolution of the pair configuration.

\section{A. Pair-Mobility and $\Phi$ Tensor}

The properties of a stokeslet object can be derived self-consistently from the linear relations which describe the stokeslets' configuration. This is done without finding the stokeslets' strengths explicitly. Below we find the pair-mobility matrix, and the $\Phi$ tensor associated 
with a single object, given the stokeslet configuration and the size of the spheres that they represent.

Each of the two objects, $x=a, b$, consists of $N_{x}$ stokeslets, $\boldsymbol{F}^{x}=\left(\vec{F}_{1}^{x}, \ldots, \vec{F}_{N_{x}}^{x}\right)$, in a known configuration, $\boldsymbol{r}^{x}=\left(\vec{r}_{1}^{x}, \ldots, \vec{r}_{N_{x}}^{x}\right)$. Here, we use the notation of a bold letter to denote a set of $N 3$-vectors, and $\vec{r}_{n}^{x}$ indicates the position 3 -vector of the $n$th stokeslet in object $x$ with respect to the object's origin. Each stokeslet is a sphere of radius $\rho$, where $\rho<\min \left(r_{1}^{x}, \ldots, r_{N_{x}}^{x}\right)$. The boundary conditions at the sphere surface enter only through its self-mobility coefficient. The velocities of the spheres, $\vec{v}_{n}^{x}$, are known from the object's linear and angular velocities,

$$
\left(\begin{array}{c}
\boldsymbol{v}^{a} \\
\boldsymbol{v}^{b}
\end{array}\right)=\left(\begin{array}{cc}
\mathbb{U}^{a} & 0 \\
0 & \mathbb{U}^{b}
\end{array}\right)\left(\begin{array}{c}
\overrightarrow{\mathcal{V}}^{a} \\
\overrightarrow{\mathcal{V}}^{b}
\end{array}\right), \quad \text { with } \mathbb{U}^{x}=\left(\begin{array}{c}
\mathbb{I}_{3 \times 3},-\vec{r}_{1}^{x \times} / l \\
\vdots \\
\mathbb{I}_{3 \times 3},-\vec{r}_{N_{x}}^{x \times} / l
\end{array}\right), \quad \text { for } x=a, b,
$$

where the matrix $\vec{y}^{\times}$obtained from the vector $\vec{y}$ is defined as $\left(\vec{y}^{\times}\right)_{i j}=\epsilon_{i k j} y_{k}$. Each stokeslet force is proportional to the relative velocity of the sphere that it represents, with respect to the flow around it as created by the other stokeslets. This gives a linear relation between the stokeslets and the velocities of the spheres ${ }^{40}$,

$$
\begin{aligned}
& \left(\begin{array}{c}
\boldsymbol{v}^{a} \\
\boldsymbol{v}^{b}
\end{array}\right)=\left(\begin{array}{cc}
\mathbb{L}^{a a} & \mathbb{L}^{a b} \\
\mathbb{L}^{a b^{T}} & \mathbb{L}^{b b}
\end{array}\right)\left(\begin{array}{c}
\boldsymbol{F}^{a} \\
\boldsymbol{F}^{b}
\end{array}\right), \\
& \left(\mathbb{L}_{n m}^{x x}\right)_{i j}= \begin{cases}\mathbb{G}_{i j}\left(\vec{r}_{n}^{x}-\vec{r}_{m}^{x}\right) & \text { if } n \neq m \\
\gamma^{-1} \delta_{i j} & \text { else }\end{cases} \\
& \left(\mathbb{L}_{n m}^{a b}\right)_{i j}=\mathbb{G}_{i j}\left(\vec{R}+\vec{r}_{n}^{a}-\vec{r}_{m}^{b}\right),
\end{aligned}
$$

and $\gamma=6 \pi \rho / l$.

First we find the pair-mobility matrix as a generalization of the analysis in Ref. 9. The sum of the stokeslets and the corresponding total torque must be equal to the external generalized forces applied on the objects. In a matrix form we can write

$$
\left(\begin{array}{c}
\overrightarrow{\mathcal{F}}^{a} \\
\overrightarrow{\mathcal{F}}^{b}
\end{array}\right)=\left(\begin{array}{cc}
\left(\mathbb{U}^{a}\right)^{T} & 0 \\
0 & \left(\mathbb{U}^{b}\right)^{T}
\end{array}\right)\left(\begin{array}{l}
\boldsymbol{F}^{a} \\
\boldsymbol{F}^{b}
\end{array}\right)
$$

Using Eqs. 12 and (13), we have

$$
\left(\begin{array}{cc}
\mathbb{U}^{a} & 0 \\
0 & \mathbb{U}^{b}
\end{array}\right)^{T} \cdot\left(\begin{array}{cc}
\mathbb{L}^{a a} & \mathbb{L}^{a b} \\
\mathbb{L}^{a b^{T}} & \mathbb{L}^{b b}
\end{array}\right)^{-1} \cdot\left(\begin{array}{cc}
\mathbb{U}^{a} & 0 \\
0 & \mathbb{U}^{b}
\end{array}\right) \cdot\left(\begin{array}{l}
\overrightarrow{\mathcal{V}}^{a} \\
\overrightarrow{\mathcal{V}}^{b}
\end{array}\right)=\left(\begin{array}{c}
\overrightarrow{\mathcal{F}}^{a} \\
\overrightarrow{\mathcal{F}}^{b}
\end{array}\right)
$$


From this expression we identify the pair-mobility matrix as

$$
\mathbb{M}=\left[\left(\begin{array}{cc}
\mathbb{U}^{a} & 0 \\
0 & \mathbb{U}^{b}
\end{array}\right)^{T} \cdot\left(\begin{array}{cc}
\mathbb{L}^{a a} & \mathbb{L}^{a b} \\
\mathbb{L}^{a b^{T}} & \mathbb{L}^{b b}
\end{array}\right)^{-1} \cdot\left(\begin{array}{cc}
\mathbb{U}^{a} & 0 \\
0 & \mathbb{U}^{b}
\end{array}\right)\right]^{-1}
$$

This expression allows to calculate the pair-mobility matrix, with the help of Eqs. (12) and (14), based on the stokeslets' configuration and the Oseen tensor alone.

Next, we derive the $\Phi^{x}$ tensor of a stokeslet object $x$. From this tensor we may readily obtain the second multipole of the pair interaction (cf. Sec. III). The force dipole around the origin of a forced object is given by [Eq. (8)], $(\boldsymbol{r} \boldsymbol{F})^{x} \equiv\left(\Phi^{x}\right)^{T} \cdot \overrightarrow{\mathcal{F}}^{x}$. Similar to the $\mathbb{U}^{x}$ matrix relating the stokeslets to the total generalized force, $\overrightarrow{\mathcal{F}}^{x}=\left(\mathbb{U}^{x}\right)^{T} \cdot \boldsymbol{F}^{x}$, we define a tensor of rank $3, \Upsilon^{x}$, which relates the stokeslet forces to the total force dipole on the object by $(\boldsymbol{r} \boldsymbol{F})^{x}=\left(\Upsilon^{x}\right)^{T} \cdot \boldsymbol{F}^{x}$. (Note that no force dipole is applied on the individual stokeslets; being arbitrarily small they possess only a force monopole.) Specifically, it is made of $N$ blocks of $3 \times 3 \times 3$, given by $\left(\Upsilon_{n}\right)_{i j s}=r_{n, s} \delta_{i j}, n=1 \ldots N, i, j, s=1,2,3$ (i.e., $r_{n, s}$ is the $s$ Cartesian coordinate of the stokeslet $n$ ). Using Eqs. (12) and (13), we have $(\boldsymbol{r} \boldsymbol{F})^{a}=\left(\Upsilon^{a}\right)^{T} \cdot\left(\mathbb{L}^{a a}\right)^{-1} \cdot \mathbb{U}^{a} \cdot \overrightarrow{\mathcal{V}}^{a}$. This implies $\left(\Phi^{x}\right)^{T}=\left(\Upsilon^{x}\right)^{T} \cdot\left(\mathbb{L}^{x x}\right)^{-1} \cdot \mathbb{U}^{x} \cdot \mathbb{M}_{\text {self }}^{x}$. Recalling that the matrices $\mathbb{M}_{\text {self }}$ and $\mathbb{L}$ are symmetric, we finally get

$$
\Phi^{x}=\mathbb{M}_{\text {self }}^{x} \cdot\left(\mathbb{U}^{x}\right)^{T} \cdot\left(\mathbb{L}^{x x}\right)^{-1} \cdot \Upsilon^{x}
$$

It is important to note that in the above derivation we compute $\mathbb{M}$ and $\Phi$ under the assumption that, for each object, the stokeslet sizes are arbitrarily small compared to the distances between them, $\rho \ll l$ (where $l$ is the object's radius of gyration). However, in a

more general case one can use the Rotne-Prager-Yamakawa tensor $\stackrel{4142}{ }$, which corrects the Oseen tensor for force distributions with finite $\operatorname{siz} \mathrm{e}^{28}$.

\section{B. Numerical Time Integration}

We present a numerical integration scheme for the dynamics of two stokeslet objects. We should first define the reference frames used in the scheme. Each rigid object is characterized by axes which are affixed and rotate with it. We define the object reference frame (ORF) such that its $z$ axis coincides with the object's alignment axis (the corresponding eigenvector of the $\mathbb{T}$-matrix). The other two axes are selected arbitrarily. The $z$ axis of the laboratory 
frame is defined along the forcing direction. During the evolution we follow the translation and rotation of the ORF in the laboratory frame.

We represent the orientation of an object by the Euler-Rodrigues 4-parameters ${ }^{43}$, defined by $(\Gamma, \vec{\Omega}) \equiv\left(\cos \frac{\theta}{2}, \hat{n} \sin \frac{\theta}{2}\right)$, where $\hat{n}$ and $\theta$ are the axis and angle of rotation $\underline{44}$. The following properties hold for this 4-parameter representation: (a) The norm of $(\Gamma, \vec{\Omega})$ in $4 \mathrm{D}$-space is unity, $\Gamma^{2}+\Omega^{2}=1$. (b) A rotation matrix is given by Rodrigues' rotation formula,

$$
R(\Gamma, \vec{\Omega})=\mathbb{I}_{3 \times 3}+2 \Gamma \vec{\Omega}^{\times}+2\left(\vec{\Omega}^{\times}\right)^{2} .
$$

(c) The parameters are invariant under inversion, i.e., $(\Gamma, \vec{\Omega})$ and $(-\Gamma,-\vec{\Omega})$ correspond to the same orientation. (d) Given the angular velocity of the object, the dynamics of its orientation simply reads

$$
\left(\begin{array}{c}
\dot{\Gamma} \\
\dot{\vec{\Omega}}
\end{array}\right)=\frac{1}{2}\left(\begin{array}{cc}
0 & -\vec{\omega}^{T} \\
\vec{\omega} & \vec{\omega}^{\times}
\end{array}\right)\left(\begin{array}{l}
\Gamma \\
\vec{\Omega}
\end{array}\right) .
$$

Since we choose the ORF such that the $z$-axis is the axis of alignment, the terminal orientation of an axially alignable object under a constant force along the $z$-axis is $(\Gamma, \vec{\Omega})=$ $\left(\cos \left(\frac{\omega t+\alpha}{2}\right), \hat{z} \sin \left(\frac{\omega t+\alpha}{2}\right)\right)$, where $\alpha$ is a constant phase which depends on the object's initial orientation at time $t=0$.

The state of a pair of objects at time $t$ is described by the position of the origins of the objects, $\vec{R}^{a}(t)$ and $\vec{R}^{b}(t)$, and their orientation parameters, $\left(\Gamma^{a}(t), \vec{\Omega}^{a}(t)\right)$ and $\left(\Gamma^{b}(t), \vec{\Omega}^{b}(t)\right)$. We time-integrate from the initial state, $\vec{R}_{0}^{a}=(0,0,0), \vec{R}_{0}^{a}-\vec{R}_{0}^{b}=\vec{R}_{0},\left(\Gamma_{0}^{a}, \vec{\Omega}_{0}^{a}\right)$ and $\left(\Gamma_{0}^{b}, \vec{\Omega}_{0}^{b}\right)$, as follows. Given the positions of the stokeslets at time $t$, the pair-mobility matrix, $\mathbb{M}(t)$, is calculated as explained in Sec. IVA, either exactly or using the multipole approximation. Then, the linear and angular velocities of the objects are given by $\left(\overrightarrow{\mathcal{V}}^{a}(t), \overrightarrow{\mathcal{V}}^{b}(t)\right)^{T}=\mathbb{M}(t)$. $\left(\overrightarrow{\mathcal{F}}^{a}(t), \overrightarrow{\mathcal{F}}^{b}(t)\right)^{T}$. From them the origins and orientations of the objects at time $t+d t$ are derived according to

$$
\begin{aligned}
\vec{R}^{x}(t+d t) & =\vec{R}^{x}(t)+\vec{V}^{x}(t) d t \\
\left(\begin{array}{l}
\Gamma^{x}(t+d t) \\
\vec{\Omega}^{x}(t+d t)
\end{array}\right) & =\exp \left[\frac{d t}{2}\left(\begin{array}{cc}
0 & -\vec{\omega}^{x T} \\
\vec{\omega}^{x} & \vec{\omega}^{x \times}
\end{array}\right)\right]\left(\begin{array}{l}
\Gamma^{x}(t) \\
\vec{\Omega}^{x}(t)
\end{array}\right)
\end{aligned}
$$

for $x=a, b$. During the evolution we make sure that the small stokeslet spheres do not overlap, and that the pair mobility matrix remains positive-definite. In practice we never encountered such problems when using the exact pair mobility matrices; when it did happen in the case of the multipole approximation we stopped the integration. 
We define a scalar order parameter which characterizes the degree of mutual alignment of the two objects,

$$
m \equiv\left[\left(\Gamma^{a}, \vec{\Omega}^{a}\right) \cdot\left(\Gamma^{b}, \vec{\Omega}^{b}\right)\right]^{2}=\left(\Gamma^{a} \Gamma^{b}+\vec{\Omega}^{a} \cdot \vec{\Omega}^{b}\right)^{2}
$$

As required, the order parameter is invariant under 3-rotation. This can be verified by explicitly applying a 3-rotation to the laboratory frame, or alternatively, by the following argument. Since 3-rotation leaves the norm of the 4-parameter orientation unchanged (property (a) above), it is a unitary transformation in 4-space. Hence, the dot product is invariant. When the objects are aligned, $\left(\Gamma^{a}, \vec{\Omega}^{a}\right)= \pm\left(\Gamma^{b}, \vec{\Omega}^{b}\right)$, and $m=1$; otherwise $0 \leq m<1$. In the case of partial alignment, $m=\cos ^{2}\left(\frac{\Delta \alpha}{2}\right)$, where $\Delta \alpha$ is the mutual phase difference 4 .

Another scalar property of the two-object system is the energy dissipation rate. At time $t$, the latter is given by $\overrightarrow{\mathcal{V}}^{a}(t) \cdot \overrightarrow{\mathcal{F}}^{a}(t)+\overrightarrow{\mathcal{V}}^{b}(t) \cdot \overrightarrow{\mathcal{F}}^{b}(t)$. Since the pair-mobility matrix is positive definite the energy dissipation of the driven pair is positive at all times.

\section{NUMERICAL RESULTS: EFFECT ON ALIGNMENT}

We present in Figs. 38 several examples for the numerically integrated evolution of object pairs under various conditions. One can be immediately appreciate the diversity of possible trajectories. To make your way through this richness it is important to make two distinctions between types of trajectories. The first distinction is between constant forcing (as in sedimentation), which can make the objects only partially aligned without synchronizing their phases of rotation ${ }^{59}$, and a time-dependent forcing protocol, which is known to lock the phase of an individual object onto that of the force ${ }^{10 \mid 11}$. The main issue examined below is how the presence of hydrodynamic interaction affects these two behaviors. The second distinction, therefore, is whether hydrodynamic interactions are included (dashed, dotted and dash-dotted/colored curves) or turned off (solid gray curves). In the absence of hydrodynamic interactions (or when they get weak as the objects move far apart), the time-dependent aligning force will make the objects fully synchronized, whereas under constant forcing the objects will generally become unaligned.

The results are presented in a dimensionless form, using units such that $\eta=\left|\omega_{0}\right|=$ 1 and $\rho=0.01$. The distances between the stokeslets of each object are taken randomly between 0 and 1 ; hence, $\rho \ll l \sim 1$. The time-dependent forcing protocol is $\vec{F}=$ 
$F_{0}\left(-\sin \left(\omega_{0} t\right) \sin (\theta), \cos \left(\omega_{0} t\right) \sin (\theta),-\cos (\theta)\right)$, where $\theta=0.1 \pi, F_{0}=-\left|\lambda_{3}\right|^{-1}, \omega_{0}=\operatorname{sign}\left(\lambda_{3}\right)$ and $\lambda_{3}$ is the real eigenvalue of the single-particle twist matrix. We examine both the trajectories of the separation vector connecting the origins of the two objects, and the corresponding evolution of the orientation order parameter.

We begin with the case of a time-dependent forcing, Figs. 3 and 4 . The first observation, most clearly demonstrated in Fig. 4(b), is that hydrodynamic interaction degrades the alignment of the two objects, as has been rigorously inferred based on symmetry considerations in Sec. IIB. Another conclusion, supported by additional examples not shown here, is that most objects, which start sufficiently far apart, especially if they start fully aligned, tend to repel each other (Fig. 3). Even if they are not fully aligned, the growing distance and weakening interaction make them individually more aligned with the forcing, and therefore also mutually synchronized. Thus, the repulsion helps restore the alignment at long times. The increasing separation occurs in the $x y$ plane, while along the $z$ axis the separation decreases and saturates to a finite distance, dependent on initial conditions, see Fig. 3.

The repulsion is accompanied by a decrease in dissipation rate (up to small oscillations), as demonstrated in Fig. 7. When the HI is turned off, the dissipation rate reaches a constant value as the two independent objects set into their ultimate aligned state (solid curves in Fig. 7).

The repulsive effect is observed for most examples of our randomly generated pairs of objects but is not a general law. For instance, when the objects start at a sufficiently small separation, some pairs remain "bound" in a limit cycle, oscillating about a certain mean separation and mean orientational alignment, as demonstrated by the green/dashed curves in Figs. 3 and 4.

In Figs. 5 and 6 we examine the same properties under constant forcing. The two effectsdegradation in the alignment of a pair which is initially fully aligned, and mutual repulsionare observed here as well. Yet, in the absence of a time-dependent aligning force, as the two objects move apart, alignment is not restored. At long times, and for the common case of repulsion, we distinguish between two observed behaviors: a) The order parameter continues to change without saturating to a constant value (e.g., red/dash-dotted curve and cyan/dotted curve in Figs. 6(a) and 6(b), respectively). This non-intuitive result can be explained as follows. The fact that the interaction becomes weak does not necessarily imply that the accumulation of phase difference stops. If the two distant objects are partially 
aligned we have $m \simeq \cos ^{2}\left(\delta \omega_{z} t / 2\right)$, where $\delta \omega_{z}$ is the difference between the objects' angular velocity along the alignment axis. Hence, if the decay of $\delta \omega_{z}$ with time is slower then $t^{-1}$ then phase difference will continue to accumulate. This depends on the detailed dynamics of repulsion which will be addressed in publication II. b) The other option is that $m$ converges to some value dependent on the initial state, with no particular chosen $m$ (green/dash-dotted curve in Fig. 6(b) and cyan/dash-dotted curve in Fig. 6(c)), i.e., the two objects continue to rotate with a fixed relative orientation. In the examples that we checked there seems to be a tendency toward ultimate anti-alignment $(m=0)$. Therefore, we also checked the stability of anti-alignment in pairs which start from such a state. Fig. 6(d) examines the stability of this configuration for objects initially confined to the $x y$ plane (perpendicular to the force). Whereas the aligned pair (blue/dotted curve) is unstable, the anti-aligned one (red/dashed curve) remains stable for the duration of integration. It may well be that this stability survives for a long but finite time, see e.g., dark red/dotted curve in Fig. 66(c). In addition, a separation of the pair along the $z$-axis destabilizes an anti-aligned pair as well (examples not shown). Finally, we note that even if the final phase difference were arbitrary and uniformly distributed, the value of $m$ would be evenly distributed around $1 / 2$ but non-uniformly, with larger weights on $m=0,1$. (This follows from the definition of $m$, see Eq. 222).)

Figure 8 compares results obtained using the full pair-mobility matrix of the stokeslet objects with those obtained from the multipole (dipole) approximation. As expected, the two calculations agree for objects whose mutual distance increases with time, and disagree for objects whose trajectories reach close proximity.

Further investigation (not shown here) of the orientational dynamics of the objects suggests a possible explanation for the characteristic repulsion between two chiral objects. In the absence of $\mathrm{HI}$, each object rotates along $\hat{F}$ and translates on average along $\hat{F}$. One contribution to the dipolar term of the HI comes from the effect on each object by the vorticity of the Oseen flow caused by the other object. This perturbative angular velocity is along an axis which is perpendicular to the separation vector and the force, $\hat{\omega}_{\text {flow }}^{a} \propto-\hat{R} \times \hat{F}$ and $\hat{\omega}_{\text {flow }}^{b} \propto \hat{R} \times \hat{F}$. The competition between this rotation and the aligning self-response of each individual object results in an inclination of the two objects relative to their non-interacting state. This inclination alters the average unperturbed linear velocity of the object by a small rotation about the $\hat{R} \times \hat{F}$ direction- counter-clockwise for object $a$ and clockwise for object $b$. Hence, the two objects glide away from each other, 

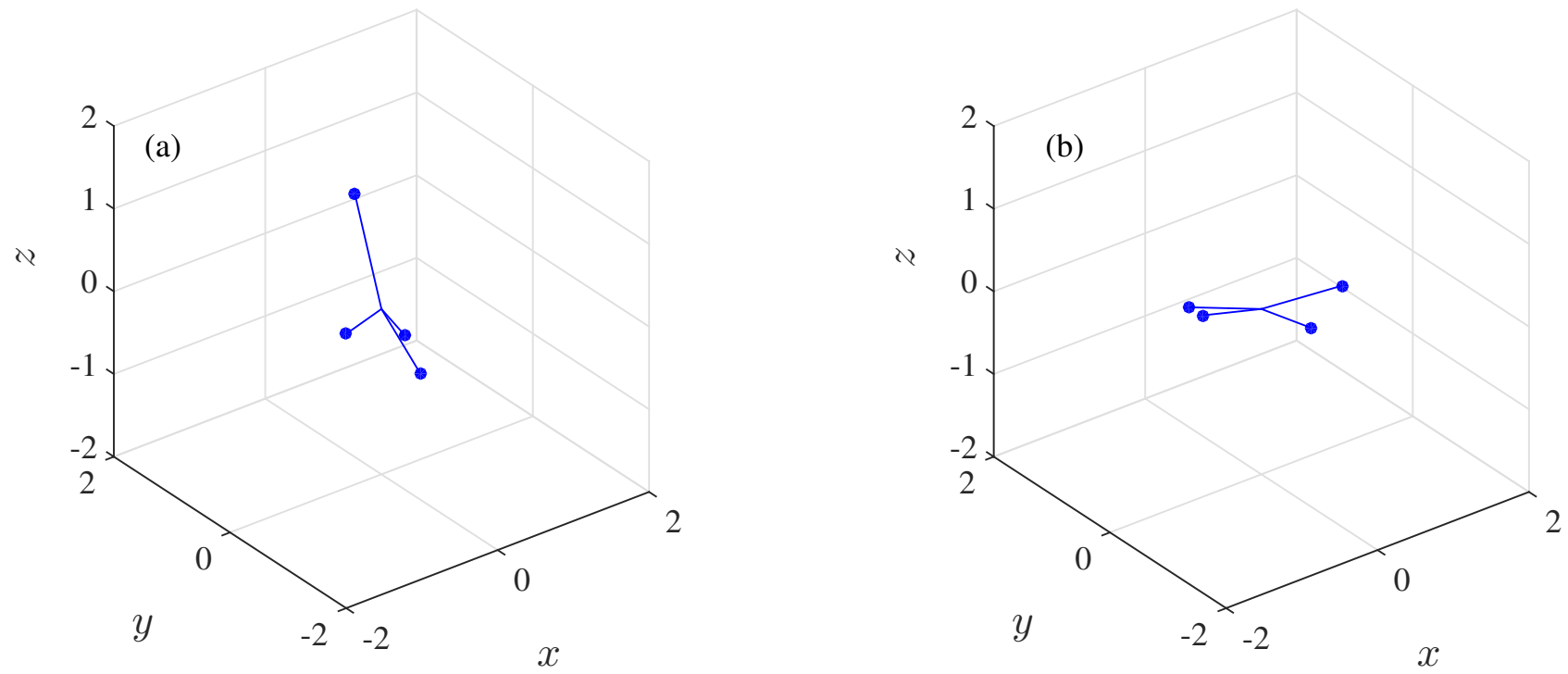

FIG. 2. Two examples of axially alignable stokeslet objects, which were used in the simulations. The objects comprise four stokeslets connected by dragless rods. The origin of the objects is at point $(0,0,0)$ and the aligning direction is $-\hat{z}$. The object on the left corresponds to the dark red/dotted trajectories in the left panels of Figs. 5 and 6, and the one on the right corresponds to the purple/dashed trajectories in the right panels of Figs. 3 and 4.

$\dot{R}^{2}=2\left(\vec{V}^{a}-\vec{V}^{b}\right) \cdot \vec{R} \propto((-\hat{R} \times \hat{F}) \times \hat{F}) \cdot \vec{R}=R\left(1-(\hat{R} \cdot \hat{F})^{2}\right) \geq 0$, where the proportionality constant is positive, i.e., the separation increases with time (unless $\vec{R} \| \vec{F}$, for which the whole argument does not hold).

\section{DISCUSSION}

Irregular objects display rich dynamics already at the level of a pair of objects, as has been demonstrated above. In the present work we have focused on the effect of the hydrodynamic interaction on the orientational alignment of such object pairs.

The hydrodynamic interaction, in general, degrades the alignment. We have rigorously proven the instantaneous linear degradation for fully aligned objects at large mutual distances. In other circumstances, such as nearby or unaligned objects, the hydrodynamic interaction may have an opposite effect. The leading degradation effect in distance is dipolar rather than monopolar; yet, it is significant - a large mutual distance (compared to the object size) is required to make the degradation negligible. More quantitatively, the 

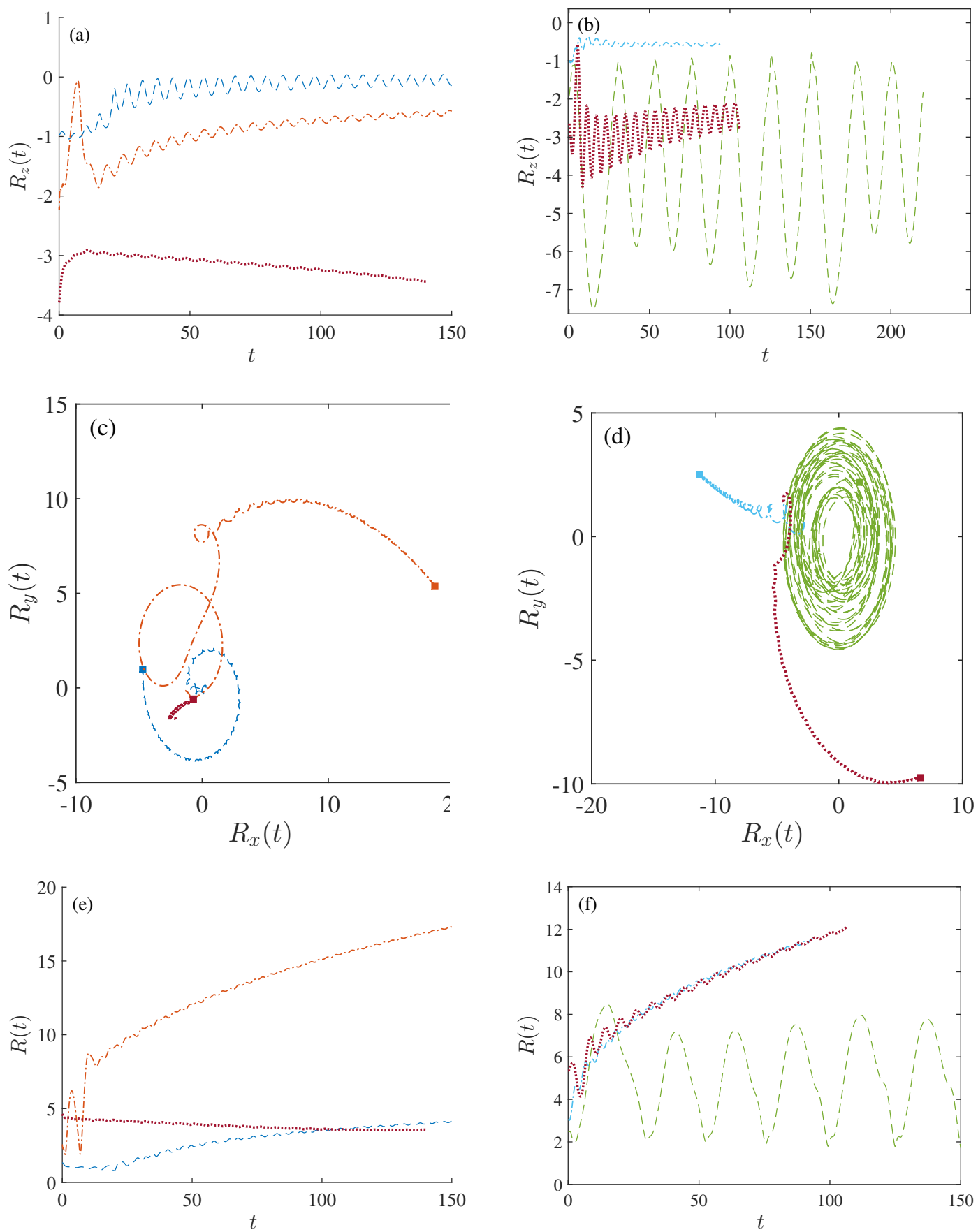

FIG. 3. Trajectories of object separation under time-dependent forcing. The three rows, from top to bottom, correspond, respectively, to the separation along the $z$ direction, its projection onto the $x y$ plane, and its total magnitude. The squares in the middle row indicate the state at the end of the simulation. The panels show results for three different objects, starting from either a random mutual orientation (left column) or their fully aligned state (right column). The green/dashed trajectory on the right panels was integrated longer than 150 time units to verify that it continues in a limit cycle. 

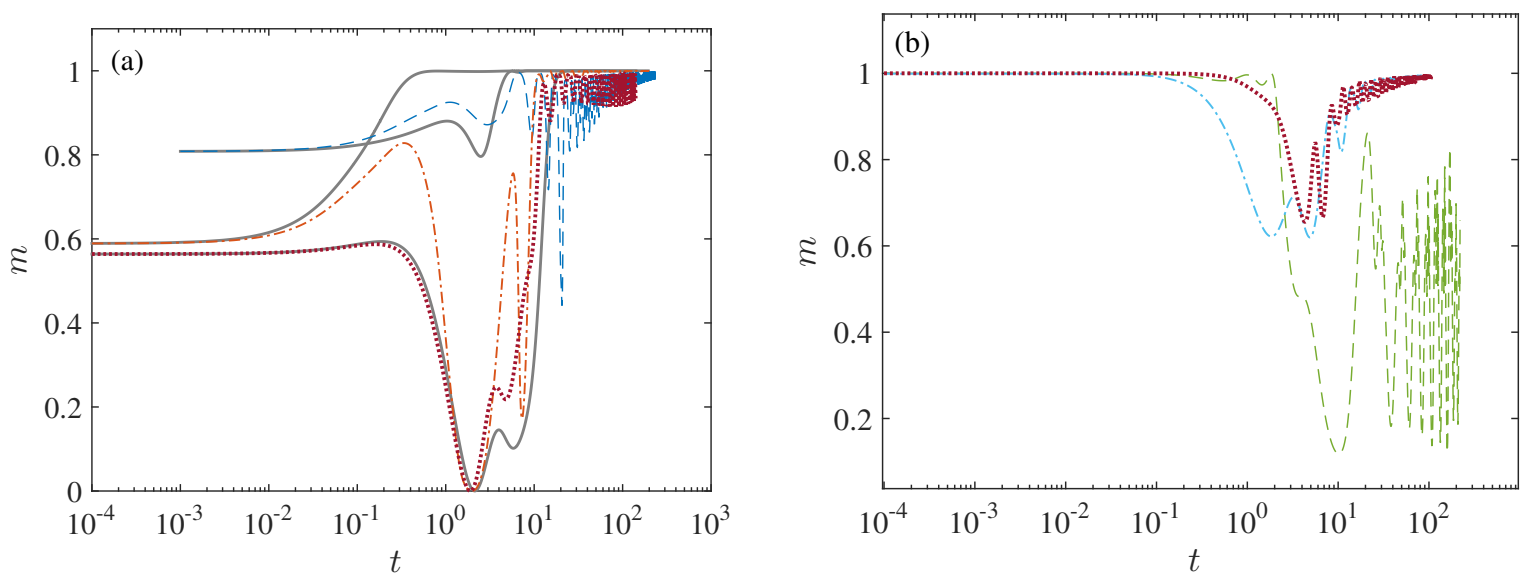

FIG. 4. Orientation order parameter as a function of time, under time-dependent forcing, for the examples of Fig. 3. (a) results for random initial orientations (examples on the left column of Fig. 3); the additional solid gray curves correspond to non-interacting objects. (b) results for initially fully aligned object pairs (right column in Fig. 3).

degradation will be significant when the perturbation to the angular velocity due to HI, $\delta \omega$, becomes comparable to the inverse of the time required to align a single object. The unperturbed angular velocity is given by $\omega_{0}=\mathbb{T}_{\text {self }} F$. The dimensionless eigenvalue of the self-twist matrix is generally found to be about an order of magnitude smaller than the dimensionless self-mobility coefficient ${ }^{8110111}$, i.e., $\omega_{0} \sim 0.1 F /\left(8 \pi l^{2}\right)$. As presented in Sec. IIB, $\delta \omega \sim \mathbb{T}^{a b} F \sim F /\left(8 \pi l^{2}\right)(l / R)^{2}$. The alignment time is typically $t_{\mathrm{al}} \sim 10 / \omega_{0}$ (see Fig 4 ). Hence, the degree of degradation is $t_{\mathrm{a} 1} \delta \omega \sim 10^{2}(l / R)^{2}$. The conclusion is that the separation between the objects should be larger than ten times their size to maintain alignment. In the case of many objects this implies a maximum volume fraction $(l / R)^{3} \sim 10^{-3}$.

At the same time, as shown in Sec. $\mathrm{V}$, for most of our randomly generated pairs of objects, the hydrodynamic interaction makes the rotating objects repel each other. As a result, at long times the hydrodynamic interaction usually becomes negligible and each of the objects gets aligned again with the time-dependent force. In that section we also provided a possible explanation for this repulsion, related to the mutual rotation of the two objects which causes them to glide away from each other. In fact, the objects need not be irregular to exhibit this gliding effect; two forced ellipsoids which start parallel to one another will experience the same repulsion20129466. The resulting hydrodynamic "pseudo-potential" will be addressed in a future publication. For the case of a finite number of objects the 

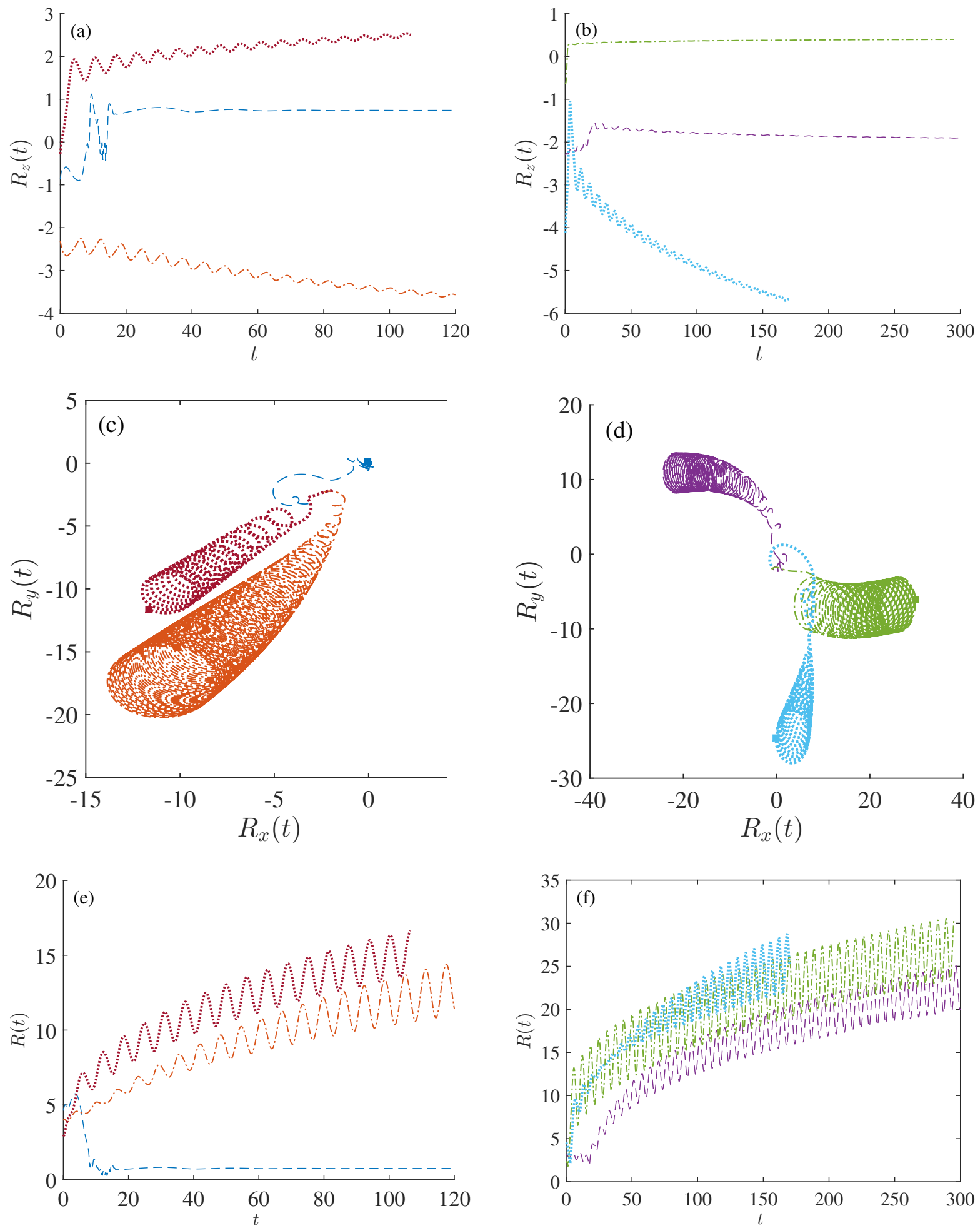

FIG. 5. Trajectories of particle separation under constant forcing. The meaning of the various panels is the same as in Fig. 3. In all the examples shown here, the two objects repel each other except of the example which corresponds to the blue/dashed curve in the left panels. (The repulsive trajectories were actually integrated to times longer than presented here.). 

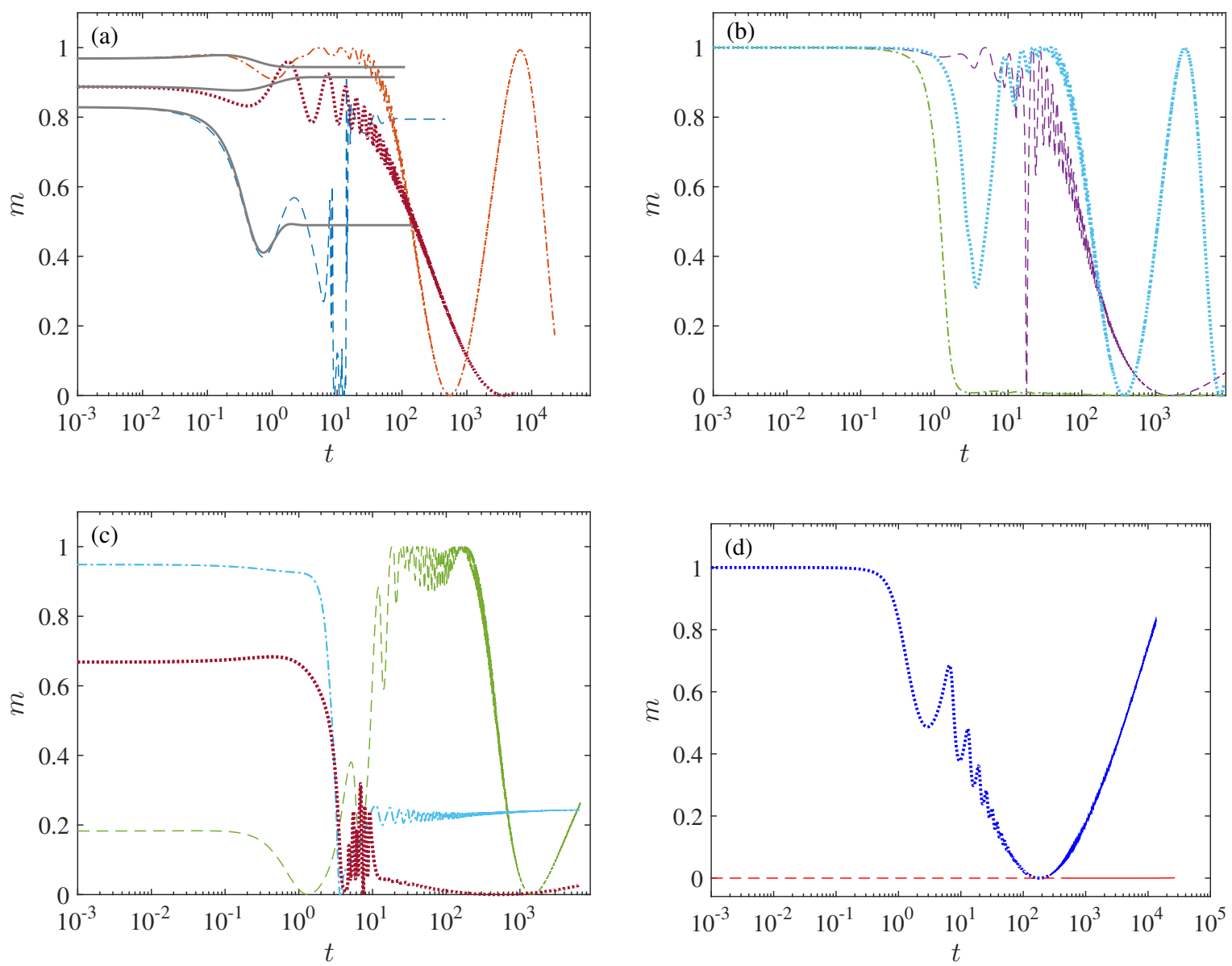

FIG. 6. Orientation order parameter as a function of time, under constant forcing, for the examples of Fig. 5. (a) results for random initial orientations (examples on the left column of Fig. 5); the solid gray curves correspond to non-interacting objects. (b) results for initially fully aligned object pairs (right column in Fig. 3). (c) results for objects with initial partial alignment (rotating around the same axis with random initial phases). (d) the stability of anti-alignment; shows trajectories of two identical pairs, which start on the $x y$ plane from the same separation and axes of rotation but with different relative phases. Blue/dotted and red/dashed curves represent, respectively, a pair which starts aligned (zero relative phase) and one which starts anti-aligned (relative phase of $\pi)$.

repulsion will help restore the alignment as the objects drift apart. It should be kept in mind, however, that the repulsion is not a general law. We observed it for a few dozens pairs of stokeslet objects. As mentioned above, it also holds for a pair of well separated ellipsoids. Yet, a few counter-examples have been also provided in Sec V. 

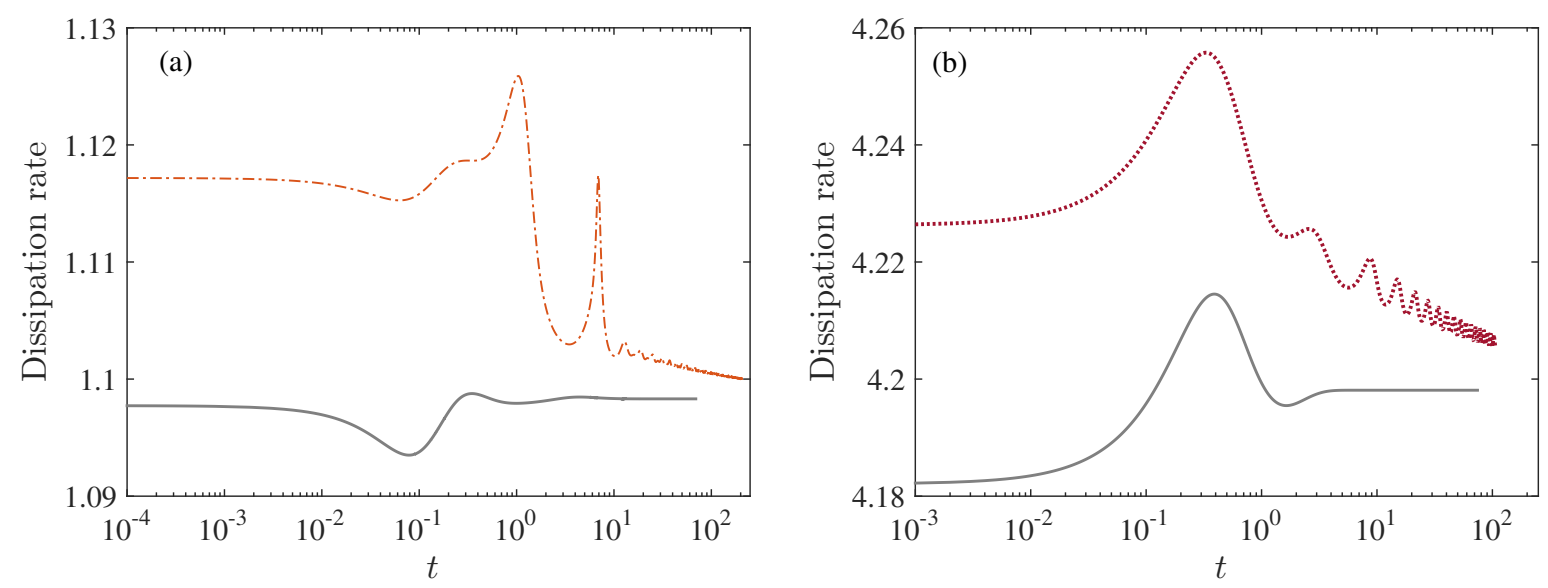

FIG. 7. Dissipation rate as a function of time for object pairs starting from arbitrary orientations, under time-dependent forcing (a) and constant forcing (b). Dash-dotted and dotted colored curves correspond to the examples of the same styles/colors in the preceding figures. Solid curves show the results in the absence of $\mathrm{HI}$.
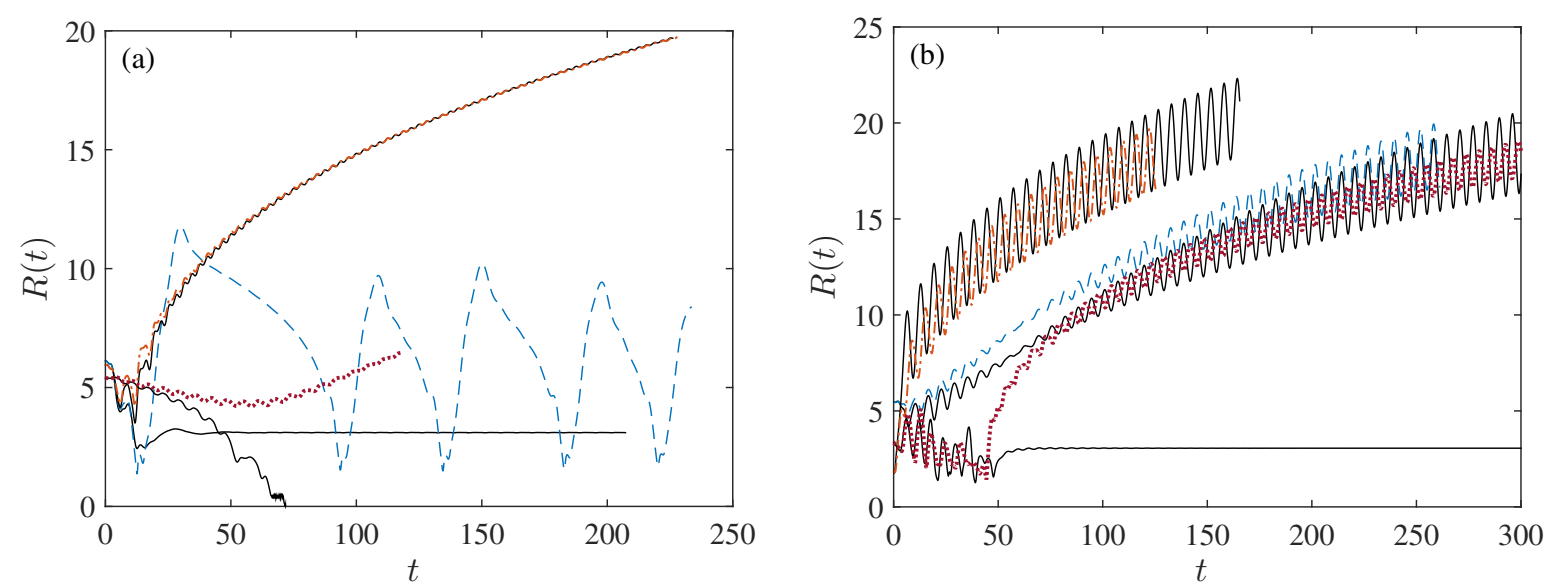

FIG. 8. Comparison between the evolution of pair separations obtained using the full pair-mobility matrix (dashed, dotted and dash-dotted colored curves) and its multipole approximation (solid curves). Each panel presents three examples of pairs under time-dependent (a) and constant forcing (b). All pairs start from a fully aligned state. The multipole approximation includes the monopolar and dipolar terms.

An interesting counterpart of the effects discussed here is found in the interaction between a forced object and a nearby wall ${ }^{1 / 48}$. The wall can be represented by an image (though not identical) object forced in the opposite direction $\underline{49}$. As a result, the object will rotate and, if it is non-spherical, also glide toward the wall, as was indeed shown for a rod falling near 


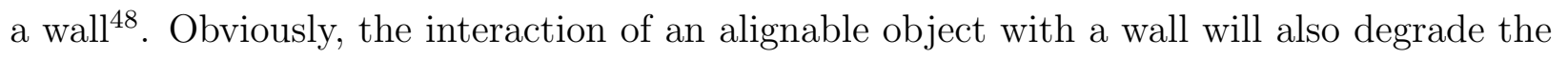
alignment.

An important distinction between regular and irregular objects, which we have not dealt with here, concerns many-body interactions in forced systems. A pair of forced spheres does not develop any relative translational velocity 1 . The same holds for a pair of forced uniform ellipsoids to order $1 / R^{3}$ (for an ellipsoid, the components of $\Phi$ which correspond to the translational velocity vanish ${ }^{[32}$ ). For a suspension of many objects this implies that two-body effects on relative motion are either absent (spheres) or negligible at low volume fraction (ellipsoids). By contrast, as we have shown here, a pair of irregular objects develops a relative velocity already at order $1 / R^{2}$, which should lead to significant two-body interactions in a suspension. This may bring about qualitative differences between driven suspensions of regular and irregular objects in relation to such phenomena as sedimentation.

This work shows that asymmetry in sedimenting objects leads to a wealth of hydrodynamic interaction effects not seen for spheres. This study was undertaken to assess how interactions disrupt the rotational synchronization of such objects. However it proves to have striking effects independent of this alignment. The prevalent repulsion, the occasional entrapment and the intricate quasiperiodic motions shown above are examples. These effects could have significant impacts on real colloidal dispersions, e.g., in fluidized beds of catalyst particles. Though we have studied only pairwise interactions between identical objects, many of these effects are expected to apply more generally. The general treatment of hydrodynamic interaction and its dependence on the shape of the interacting objects, which we have developed here, should prove useful in exploring these phenomena. Our work in progress aims to achieve a more general understanding of the rich behavior reported in Sec. V

\section{ACKNOWLEDGMENTS}

We thank Robert Deegan and Alex Leshansky for helpful discussions, and the James Franck Institute and Tel Aviv University for their hospitality during part of this work. This research has been supported by the US-Israel Binational Science Foundation (Grant no. 2012090). 


\section{Appendix A: Notation}

The dynamics of arbitrarily shaped objects is complex and requires an elaborate notation. We use the following notation regarding vectors, tensors, and matrices:

1. 3-vectors are denoted by an arrow, $\vec{v}$, and unit 3-vectors by a hat, $\hat{v}$.

2. 6-vectors are denoted by a calligraphic font, $\overrightarrow{\mathcal{F}}$.

3. Matrices are marked by a blackboard-bold letter, e.g., $\mathbb{M}$, where the dimension of the matrix is understood from the context.

4. Tensors of rank 3 are denoted by a capital Greek letter, e.g., $\Phi$.

5. A set of $N$ 3-vectors, representing $N$ stokeslets, is denoted by a bold letter, e.g., $\boldsymbol{v}^{a}=\left(\vec{v}_{1}^{a}, \ldots, \vec{v}_{N}^{a}\right)$.

6. Subscripts with parentheses, e.g., $\mathbb{M}_{(2)}$, represent a term in a multipole expansion.

7. $\mathbb{I}_{n \times n}$ is the $n \times n$ identity matrix.

8. Tensor multiplication - the dot notation, - - denotes a contraction over one index. The double dot notation, :, denotes a contraction over two indices. Thus, given a tensor $\Upsilon$ of rank $N$ and a tensor $\Xi$ of $\operatorname{rank} M>N$, the tensors $\Upsilon \cdot \Xi$ and $\Upsilon: \Xi$ are tensors of rank $N+M-2$ and $N+M-4$. For example, for $\Upsilon$ of rank 2 and $\Xi$ of $\operatorname{rank} 3,(\Xi \cdot \Upsilon)_{i k j}=\Upsilon_{i s} \Xi_{s k j}$ and $(\Upsilon: \Xi)_{j}=\Upsilon_{k s} \Xi_{s k j}$

9. The matrix $\vec{Y}^{\times}$obtained from the vector $\vec{Y}$ is defined as $\left(\vec{Y}^{\times}\right)_{i j}=\epsilon_{i k j} Y_{k}$, such that, for any vector $\vec{X}, \vec{Y} \times \vec{X}=\vec{Y} \times \vec{X}$.

\section{Appendix B: Pair-mobility: Change of object origin}

Here we derive the transformation of the pair-mobility matrix under change of objects' origins. Consider a new choice of origins given by $\vec{R}^{a \prime}=\vec{R}^{a}+\vec{h}^{a}$ and $\vec{R}^{b \prime}=\vec{R}^{b}+\vec{h}^{b}$, and denote the objects' properties with respect to the new origins with '. Following Ref. 9, the transformations for the generalized velocities and forces can be written as $\overrightarrow{\mathcal{V}}^{\prime \prime}=\left[\mathbb{I}_{6 \times 6}-\right.$ 
$\left.\left(\mathbb{B}^{x}\right)^{T}\right] \overrightarrow{\mathcal{V}}^{x}$ and $\overrightarrow{\mathcal{F}}^{x \prime}=\left[\mathbb{I}_{6 \times 6}+\mathbb{B}^{x}\right] \overrightarrow{\mathcal{F}}^{x}$ for $x=a, b$, where

$$
\mathbb{B}^{a}=\left(\begin{array}{cc}
0 & 0 \\
-\vec{h}^{a \times} & 0
\end{array}\right) \text { and } \mathbb{B}^{b}=\left(\begin{array}{cc}
0 & 0 \\
-\vec{h}^{b \times} & 0
\end{array}\right) .
$$

Using $\left[\mathbb{I}_{6 \times 6}+\mathbb{B}^{x}\right]^{-1}=\left[\mathbb{I}_{6 \times 6}-\mathbb{B}^{x}\right]$ we have

$$
\left(\begin{array}{ll}
\mathbb{M}^{\prime a a} & \mathbb{M}^{\prime a b} \\
\mathbb{M}^{\prime b a} & \mathbb{M}^{\prime b b}
\end{array}\right)=\left(\begin{array}{cc}
{\left[\mathbb{I}_{6 \times 6}-\left(\mathbb{B}^{a}\right)^{T}\right]} & 0 \\
0 & {\left[\mathbb{I}_{6 \times 6}-\left(\mathbb{B}^{b}\right)^{T}\right]}
\end{array}\right)\left(\begin{array}{cc}
\mathbb{M}^{a a} & \mathbb{M}^{a b} \\
\mathbb{M}^{b a} & \mathbb{M}^{b b}
\end{array}\right)\left(\begin{array}{cc}
{\left[\mathbb{I}_{6 \times 6}-\mathbb{B}^{a}\right]} & 0 \\
0 & {\left[\mathbb{I}_{6 \times 6}-\mathbb{B}^{b}\right]}
\end{array}\right)
$$

\section{Appendix C: Properties of the tensor $\Phi$}

Below we provide a more detailed discussion regarding the tensor $\Phi$ introduced in Sec. III] We consider its symmetries and its dependence on the choice of origin. We separate $\Phi$ into a translational part - linear velocity response to a flow gradient, denoted by $\Phi_{\text {tran }}$, and a rotational part - angular velocity response to a flow gradient, denoted by $\Phi_{\text {rot }}$. We show that $\Phi_{\text {tran }}$ is symmetric with respect to its last two indices while $\Phi_{\text {rot }}$ has also an antisymmetric part which is the Levi-Civita tensor. In addition, we show that $\Phi_{\text {tran }}$ depends on the choice of the object's origin whereas $\Phi_{\text {rot }}$ does not, and derive the transformation of the former under change of origins.

In order to prove the symmetry properties of $\Phi$ we consider its transpose tensor $\Phi^{T}=\tilde{\Phi}$ which gives the force dipole around the object when subjected to external forcing, $(\boldsymbol{r} \boldsymbol{F})=$ $\tilde{\Phi} \cdot \overrightarrow{\mathcal{F}}=\tilde{\Phi}_{\text {tran }} \cdot \vec{F}+\tilde{\Phi}_{\text {rot }} \cdot \vec{\tau}$. We write the force dipole as a sum of symmetric and antisymmetric terms, $\frac{1}{2}\left[(\boldsymbol{r} \boldsymbol{F})+(\boldsymbol{r} \boldsymbol{F})^{T}+\epsilon \cdot \vec{\tau}\right]=\tilde{\Phi}_{\text {tran }} \cdot \vec{F}+\tilde{\Phi}_{\text {rot }} \cdot \vec{\tau}$, where $\epsilon$ is the Levi-Civita tensor. The last equality implies that $\left(\tilde{\Phi}_{\text {tran }}\right)_{s k i}$ is symmetric with respect to $s$ and $k$ and that the anti-symmetric part of $\left(\tilde{\Phi}_{\text {rot }}\right)_{s k i}$ is $\frac{1}{2} \epsilon_{s k i}$.

Next we consider the transformation of $\Phi$ under change of origins. Let us assume that an object is given in a constant, arbitrary shear flow $\vec{u}(\vec{r})=S \cdot \vec{r}$, where $S$ is not necessarily a symmetric matrix. The object's linear velocities measured about $\vec{R}$ and $\vec{R}^{\prime}=\vec{R}+\vec{h}$ are $\vec{V}=S \cdot \vec{R}+\Phi_{\text {tran }}: S$ and $\vec{V}^{\prime}=S \cdot(\vec{R}+\vec{h})+\Phi_{\text {tran }}^{\prime}: S$ respectively. The tensor $\Phi_{\text {rot }}$ does not depend on the choice of origin since the angular velocity of the object is independent of that choice, $\vec{\omega}=\Phi_{\text {rot }}: S=\Phi_{\text {rot }}^{\prime}: S$. Using the relation $\vec{V}^{\prime}=\vec{V}-\vec{h} \times \vec{\omega}$ we find

$$
\Phi_{\text {tran }}^{\prime}: S=\left(\Phi_{\text {tran }}-\vec{h}^{\times} \cdot \Phi_{\text {rot }}\right): S-S \cdot \vec{h}
$$


In general, with analogy to Eq. (B.1), we can write

$$
\Phi^{\prime}=\left[\mathbb{I}_{6 \times 6}-(\mathbb{B})^{T}\right] \cdot \Phi+\Delta,
$$

where

$$
\mathbb{B}=\left(\begin{array}{cc}
0 & 0 \\
-\vec{h}^{\times} & 0
\end{array}\right) \text { and } \Delta_{i k s}=\left\{\begin{array}{cc}
-\delta_{i s} h_{k} & , i=1 \ldots 3 \\
0 \quad, i=4 \ldots 6
\end{array}\right.
$$

\section{Appendix D: Proofs of general properties of interaction multipoles}

Here we prove the two general results presented in Sec. III concerning the interaction multipoles.

Multipole expansions are constructed by repeated projections ("reflections"), between the two objects, of the Green's function and its derivatives. The self-blocks of the mobility matrix result from even projections, and the coupling blocks from odd projections. In our case $\mathbb{G}$, the Oseen tensor, has even parity and scales as $1 / R$.

The Green's function $\mathbb{G}$ itself appears only once in the expansion, in the first $(1 / R)$ multipole. This is because the force monopoles acting on the particles are prescribed. This monopolar (odd) interaction appears only in the coupling blocks. The leading multipole appearing in the self-blocks is constructed by projecting the induced force dipole on object 2 (proportional to $\nabla \mathbb{G}$ ) back onto object 1 (by another $\nabla \mathbb{G}$ ). Thus, this leading multipole is of 4 th order, proportional to $1 / R^{4}$. This proves the first result in Sec. III. Its particular manifestation for two spheres is well known ${ }^{1}$.

Now, consider the $n$th multipole, proportional to $1 / R^{n}$. Assume that it contains $k \mathbb{G}$ 's and $n-k$ derivatives. Its parity is $(-1)^{n-k}$. As explained above, for self-blocks $k$ is even, and for coupling blocks it is odd. Hence, the parity of the $n$th multipole is $(-1)^{n}$ in the self-blocks and $(-1)^{n+1}$ in the coupling blocks. This proves the second result.

\section{Appendix E: General Form of $\mathbb{M}_{(2)}^{a b}$}

Below we provide a general form of the matrix $\mathbb{M}_{(2)}^{a b}$, the 2nd-order multipole of the coupling block in the pair-mobility matrix, and point out the number of its independent components. This is done by decomposing the tensors $\Phi$ and $\Theta$ to their symmetric and anti-symmetric parts. Without loss of generality we choose the separation vector between 
the two objects to be along the $x$ axis, $\hat{R}=\hat{x}$. For two not necessarily identical objects the matrix $\mathbb{M}_{(2)}^{a b}$ has the form

$$
\left.\mathbb{M}_{(2)}^{a b}=\left(\frac{l}{R}\right)^{2}\left(\begin{array}{ccc}
A_{x x}^{a}-A_{x x}^{b}-A_{y x}^{b}-A_{z x}^{b} \\
A_{y x}^{a} & 0 & 0 \\
A_{z x}^{a} & 0 & 0
\end{array}\right)\left(\begin{array}{ccc}
-T_{x x}^{b} & -T_{y x}^{b} & -T_{z x}^{b} \\
0 & 0 & 1 \\
0 & -1 & 0
\end{array}\right)\right)
$$

where the $A_{i j}^{x}$ and $T_{i j}^{x}$ are functions of $\hat{R}$ and the shape and orientation of object $x,(x=a, b)$. For two identical (in shape and orientation) objects we have

$$
\mathbb{M}_{(2)}^{a b}=\left(\frac{l}{R}\right)^{2}\left(\begin{array}{c}
\left(\begin{array}{ccc}
0 & -A_{y x} & -A_{z x} \\
A_{y x} & 0 & 0 \\
A_{z x} & 0 & 0
\end{array}\right) \\
\left(\begin{array}{ccc}
T_{x x} & 0 & 0 \\
T_{y x} & 0 & 1 \\
T_{z x} & -1 & 0
\end{array}\right)
\end{array}\right) .
$$

\section{REFERENCES}

${ }^{1} \mathrm{~J}$. Happel and H. Brenner, Low Reynolds number hydrodynamics: with special applications to particulate media (Martinus Nijhoff, The Hague, 1983).

${ }^{2}$ W. B. Russel, D. A. Saville, and W. R. Schowalter, Colloidal Dispersions (Cambridge University Press, 1989).

${ }^{3}$ S. Ramaswamy, "Issues in the statistical mechanics of steady sedimentation," Adv. Phys. 50, 297 (2001).

${ }^{4}$ M. Makino and M. Doi, "Sedimentation of a particle with translation-rotation coupling," J. Phys. Soc. Jpn. 72, 2699 (2003).

${ }^{5}$ O. Gonzalez, A. B. A. Graf, and J. H. Maddocks, "Dynamics of a rigid body in a Stokes fluid," J. Fluid Mech. 519, 133 (2004). 
${ }^{6}$ M. Doi and M. Makino, "Motion of micro-particles of complex shape," Prog. Polym. Sci. 30, 876 (2005), plenary Lectures World Polymer Congress, 40th IUPAC International Symposium on Macromolecules.

${ }^{7}$ M. Makino and M. Doi, "Migration of twisted ribbon-like particles in simple shear flow," Phy. Fluids 17, 103605 (2005).

${ }^{8}$ K. I. Morozov and A. M. Leshansky, "The chiral magnetic nanomotors," Nanoscale 6, 1580 (2014).

${ }^{9}$ N. W. Krapf, T. A. Witten, and N. C. Keim, "Chiral sedimentation of extended objects in viscous media," Phys. Rev. E 79, 056307 (2009).

${ }^{10}$ B. Moths and T. A. Witten, "Full alignment of colloidal objects by programed forcing," Phys. Rev. Lett. 110, 028301 (2013).

${ }^{11}$ B. Moths and T. A. Witten, "Orientational ordering of colloidal dispersions by application of time-dependent external forces," Phys. Rev. E 88, 022307 (2013).

${ }^{12}$ H. Brenner, "The Stokes resistance of an arbitrary particle II: An extension," Chem. Eng. Sci. 19, 599 (1964).

${ }^{13} \mathrm{H}$. Brenner and M. E. O’Neill, “On the Stokes resistance of multiparticle systems in a linear shear field," Chem. Eng. Sci. 27, 1421 (1972).

${ }^{14}$ A. Goldman, R. Cox, and H. Brenner, "The slow motion of two identical arbitrarily oriented spheres through a viscous fluid," Chem. Eng. Sci. 21, 1151 (1966).

${ }^{15}$ S. Wakiya, "Mutual interaction of two spheroids sedimenting in a viscous fluid," J. Phys. Soc. Jpn 20, 1502 (1965).

${ }^{16}$ B. Felderhof, "Hydrodynamic interaction between two spheres," Physica A 89, 373 (1977).

${ }^{17}$ D. J. Jeffrey and Y. Onishi, "Calculation of the resistance and mobility functions for two unequal rigid spheres in low-Reynolds-number flow," J. Fluid Mech. 139, 261 (1984).

${ }^{18}$ W. Liao and D. A. Krueger, "Multipole expansion calculation of slow viscous flow about spheroids of different sizes," J. Fluid Mech. 96, 223 (1980).

${ }^{19}$ S. Kim, "Sedimentation of two arbitrarily oriented spheroids in a viscous fluid," Int. J. Multiphas. Flow 11, 699 (1985).

${ }^{20} \mathrm{~S}$. Kim, "Singularity solutions for ellipsoids in low-Reynolds-number flows: With applications to the calculation of hydrodynamic interactions in suspensions of ellipsoids," Int. J. Multiphas. Flow 12, 469 (1986). 
${ }^{21}$ E. J. Hinch and L. G. Leal, "The effect of brownian motion on the rheological properties of a suspension of non-spherical particles," Journal of Fluid Mechanics 52, 683 (1972).

${ }^{22} \mathrm{H}$. Brenner, "Rheology of a dilute suspension of axisymmetric brownian particles," International Journal of Multiphase Flow 1, 195 (1974).

${ }^{23}$ I. L. Claeys and J. F. Brady, "Suspensions of prolate spheroids in stokes flow. part 2. statistically homogeneous dispersions," Journal of Fluid Mechanics 251, 443 (1993).

${ }^{24}$ G. B. Jeffery, "The motion of ellipsoidal particles immersed in a viscous fluid," Proceedings of the Royal Society of London A102, 161 (1922).

${ }^{25}$ R. H. Davis, "Sedimentation of axisymmetric particles in shear flows," Physics of Fluids A: Fluid Dynamics (1989-1993) 3, 2051 (1991).

${ }^{26}$ S. J. Karrila, Y. O. Fuentes, and S. Kim, "Parallel computational strategies for hydrodynamic interactions between rigid particles of arbitrary shape in a viscous fluid," J. Rheol. 33, 913 (1989).

${ }^{27}$ T. Tran-Cong and N. Phan-Thien, "Stokes problems of multiparticle systems: A numerical method for arbitrary flows," Phys. Fluids A-Fluid 1, 453 (1989).

${ }^{28}$ B. Carrasco and J. G. de la Torre, "Hydrodynamic properties of rigid particles: Comparison of different modeling and computational procedures," Biophys. J. 76, 3044 (1999).

${ }^{29}$ R. Kutteh, "Rigid body dynamics approach to stokesian dynamics simulations of nonspherical particles," J. Chem. Phys. 132, 174107 (2010).

${ }^{30}$ B. Cichocki, B. U. Felderhof, K. Hinsen, E. Wajnryb, and J. Bławzdziewicz, "Friction and mobility of many spheres in Stokes flow," J. Chem. Phys. 100, 3780 (1994).

${ }^{31} \mathrm{H}$. Brenner, "The Stokes resistance of an arbitrary particle," Chem. Eng. Sci. 18, 1 (1963).

${ }^{32} \mathrm{H}$. Brenner, "The Stokes resistance of an arbitrary particle IV: Arbitrary fields of flow," Chem. Eng. Sci. 19, 703 (1964).

${ }^{33}$ D. W. Condiff and J. S. Dahler, "Brownian motion of polyatomic molecules: The coupling of rotational and translational motions," J. Chem. Phys. 44, 3988 (1966).

${ }^{34}$ L. Landau and E. Lifshitz, Statistical Physics, Part 1 (third edition) (Pergamon Press, 1980).

${ }^{35}$ Parity does not mean here symmetry under full spatial inversion, as such an operation would turn the chiral objects into their enantiomers; rather, we mean here symmetry under the inversion of $\vec{R}$. 
${ }^{36}$ T. M. Squires, "Effective pseudo-potentials of hydrodynamic origin," J. Fluid Mech. 443, $403(2001)$.

${ }^{37}$ In fact, these results are not special to the hydrodynamic interaction but can be similarly proven for any multipole expansion. As such, they were most probably derived before.

${ }^{38}$ These tensors are related to the two introduced by Brenner ${ }^{32}$. Brenner's tensors give the force and torque exerted on an object in linear response to a flow gradient in which it is embedded. Our $\Phi$ is related to these two via the individual self-mobility matrix.

${ }^{39}$ S. Kim and S. J. Karrila, Microhydrodynamics: Principles and Selected Applications (Mineola, N.Y. : Dover Publications, 2005).

${ }^{40}$ More explicitly, consider the stokeslet at position $\vec{r}_{n}^{a}$. The flow at that point which is created by the other stokeslets, belonging to the two objects, is $\vec{u}\left(\vec{r}_{n}^{a}\right)=\Sigma_{m \neq n} \mathbb{G}\left(\vec{r}_{n}^{a}-\vec{r}_{m}^{a}\right)$. $\vec{F}_{m}^{a}+\Sigma_{m} \mathbb{G}\left(\vec{R}+\vec{r}_{n}^{a}-\vec{r}_{m}^{b}\right) \cdot \vec{F}_{m}^{b}$. The stokeslet at that point is proportional to the velocity of the sphere relative to the local flow, $\vec{F}_{n}^{a}=\gamma\left(\vec{v}_{n}^{a}-\vec{u}\left(\vec{r}_{n}^{a}\right)\right)$. This gives Eq. (13).

${ }^{41}$ J. Rotne and S. Prager, "Variational treatment of hydrodynamic interaction in polymers," J. Chem. Phys. 50, 4831 (1969).

${ }^{42} \mathrm{H}$. Yamakawa, "Transport properties of polymer chains in dilute solution: Hydrodynamic interaction," J. Chem. Phys. 53, 436 (1970).

${ }^{43}$ L. D. Favro, "Theory of the rotational brownian motion of a free rigid body," Phys. Rev. 119, 53 (1960).

${ }^{44}$ This is the same as the unit-quaternion representation $\underline{43}$.

${ }^{45}$ If the symmetry of the objects is such that their phase difference is unobservable (e.g., two ellipsoids rotating around their major axis), then we set it to zero.

${ }^{46}$ I. L. Claeys and J. F. Brady, "Suspensions of prolate spheroids in stokes flow. part 1. dynamics of a finite number of particles in an unbounded fluid," Journal of Fluid Mechanics 251, 411 (1993).

${ }^{47}$ T. Squires and M. Brenner, "Like-charge attraction and hydrodynamic interaction," Phys. Rev. Lett. 85, 4976 (2000).

${ }^{48}$ W. B. Russel, E. J. Hinch, L. G. Leal, and G. Tieffenbruck, "Rods falling near a vertical wall," Journal of Fluid Mechanics 83, 273 (1977).

${ }^{49}$ C. Pozrikidis, Boundary Integral and Singularity Methods for Linearized Viscous Flow (Cambridge University Press, 1992). 